\title{
Emergence of methicillin resistance predates the clinical use of antibiotics
}

https://doi.org/10.1038/s41586-021-04265-w

Received: 12 May 2021

Accepted: 18 November 2021

Published online: 5 January 2022

\section{Open access}

Check for updates

\author{
Jesper Larsen ${ }^{1,55 \bowtie}$, Claire L. Raisen ${ }^{2,55}$, Xiaoliang Ba ${ }^{2}$, Nicholas J. Sadgrove ${ }^{3}$, \\ Guillermo F. Padilla-González ${ }^{3}$, Monique S. J. Simmonds ${ }^{3}$, Igor Loncaric ${ }^{4}$, \\ Heidrun Kerschner ${ }^{5}$, Petra Apfalter ${ }^{5}$, Rainer Hartt ${ }^{5}$, Ariane Deplano ${ }^{6}$, \\ Stien Vandendriessche ${ }^{6,46}$, Barbora Černá Bolfíková7, Pavel Hulva ${ }^{8,9}$, Maiken C. Arendrup", \\ Rasmus K. Hare', Céline Barnadas ${ }^{1,10}$, Marc Stegger', Raphael N. Sieber', Robert L. Skov" , \\ Andreas Petersen', Øystein Angen', Sophie L. Rasmussen ${ }^{12,13}$, Carmen Espinosa-Gongora ${ }^{14}$, \\ Frank M. Aarestrup ${ }^{15}$, Laura J. Lindholm ${ }^{16}$, Suvi M. Nykäsenoja ${ }^{17}$, Frederic Laurent ${ }^{18}$, \\ Karsten Becker $^{19}$, Birgit Walther ${ }^{20,47}$, Corinna Kehrenberg ${ }^{21}$, Christiane Cuny ${ }^{22}$, \\ Franziska Layer ${ }^{22}$, Guido Werner ${ }^{22}$, Wolfgang Witte ${ }^{22}$, Ivonne Stamm ${ }^{23}$, Paolo Moroni ${ }^{24,48}$, \\ Hannah J. Jørgensen ${ }^{25}$, Hermínia de Lencastre ${ }^{26,27}$, Emilia Cercenado ${ }^{28}$, \\ Fernando García-Garrote ${ }^{28,49}$, Stefan Börjesson ${ }^{29,50}$, Sara Hæggman ${ }^{30}$, Vincent Perreten ${ }^{31}$, \\ Christopher J. Teale ${ }^{32}$, Andrew S. Waller ${ }^{33,51,52}$, Bruno Pichon ${ }^{34}$, Martin D. Curran ${ }^{35}$, \\ Matthew J. Ellington ${ }^{35,53}$, John J. Welch ${ }^{36}$, Sharon J. Peacock ${ }^{37}$, David J. Seilly ${ }^{2}$, \\ Fiona J. E. Morgan ${ }^{2,54}$, Julian Parkhill' ${ }^{2}$, Nazreen F. Hadjirin ${ }^{2}$, Jodi A. Lindsay ${ }^{38}$, \\ Matthew T. G. Holden ${ }^{39}$, Giles F. Edwards ${ }^{40}$, Geoffrey Foster ${ }^{41}$, Gavin K. Paterson ${ }^{42}$, \\ Xavier Didelot ${ }^{43}$, Mark A. Holmes ${ }^{2,56}$, Ewan M. Harrison ${ }^{37,44,45,56}$ \& Anders R. Larsen ${ }^{1,56}$
}

\begin{abstract}
The discovery of antibiotics more than 80 years ago has led to considerable improvements in human and animal health. Although antibiotic resistance in environmental bacteria is ancient, resistance in human pathogens is thought to be a modern phenomenon that is driven by the clinical use of antibiotics ${ }^{1}$. Here we show that particular lineages of methicillin-resistant Staphylococcus aureus-a notorious human pathogen-appeared in European hedgehogs in the pre-antibiotic era. Subsequently, these lineages spread within the local hedgehog populations and between hedgehogs and secondary hosts, including livestock and humans. We also demonstrate that the hedgehog dermatophyte Trichophyton erinacei produces two $\beta$-lactam antibiotics that provide a natural selective environment in which methicillin-resistant $S$. aureus isolates have an advantage over susceptible isolates. Together, these results suggest that methicillin resistance emerged in the pre-antibiotic era as a co-evolutionary adaptation of $S$. aureus to the colonization of dermatophyte-infected hedgehogs. The evolution of clinically relevant antibiotic-resistance genes in wild animals and the connectivity of natural, agricultural and human ecosystems demonstrate that the use of a One Health approach is critical for our understanding and management of antibiotic resistance, which is one of the biggest threats to global health, food security and development.
\end{abstract}

Methicillin-resistant $S$. aureus (MRSA) is one of the most common antibiotic-resistant bacterial pathogens, causing approximately 171,000 invasive infections each year in Europe alone ${ }^{2}$. MRSA was first identified in 1960 shortly after the introduction of methicillin (celbenin) as a treatment option against penicillin-resistant $S$. aureus clones $^{3}$, but was possibly selected for by the clinical use of penicillin over the previous 20 years ${ }^{4}$. Methicillin resistance has subsequently emerged in many $S$. aureus clones around the world, both in hospital and community settings as well as in livestock such as pigs and cattle ${ }^{5,6}$. This has serious implications for the treatment of severe infections and the World Health Organization now considers MRSA to be an important threat to human health ${ }^{7}$.
Methicillin resistance in S. aureus is mediated by the mecA and mecC genes, which encode the enzymes penicillin-binding protein 2a (PBP2a) and PBP2c, respectively. $m e c A$ and $m e c C$ confer resistance to almost all $\beta$-lactam antibiotics, including penicillinase-labile penicillins (such as penicillin G), penicillinase-stable penicillins (such as methicillin) and cephalosporins (such as cefoxitin).

Hedgehog surveys from Denmark and Sweden demonstrated a surprisingly high prevalence of MRSA carrying $\operatorname{mecC}(\operatorname{mecC} \text {-MRSA })^{8,9}$, raising the possibility that the evolution of these bacteria was driven by natural selection in wildlife, as opposed to clinical use of antibiotics. Historically, mecC-MRSA was first discovered in dairy cows and subsequently in humans ${ }^{10}$, suggesting that the use of 
antibiotics in livestock was providing a selective advantage and that human infections were the result of zoonotic transmission. Studies from many different European countries revealed that mecC-MRSA is also present in other domesticated animals such as sheep, goats and horses as well as in a broad range of wild animals, albeit at low frequencies ${ }^{11}$.

Our hypothesis that the evolution of mecC-MRSA was driven by natural selection is supported by studies from northwestern Europe and New Zealand that showed that hedgehogs are frequently colonized with the dermatophyte $T$. erinacei, which produces a penicillinase-labile penicillin-like substance that was recently identified as penicillin $\mathrm{G}^{12-19}$. To test our hypothesis, we examined the distribution of mecC-MRSA and other S. aureus isolates in hedgehogs in ten European countries and New Zealand. We sequenced 244 S. aureus isolates from hedgehogs and 913 S. aureus isolates from other sources to infer the evolutionary histories, host dynamics, geographical dispersal patterns and zoonotic potential of the major mecC-MRSA clones in Europe. The potential mechanisms for the natural selection of mecC-MRSA by $T$. erinacei were assessed by analysing the genome of $T$. erinacei for $\beta$-lactam biosynthetic genes and by screening $T$. erinacei for the production of $\beta$-lactams and antibiotic activity against a panel of $S$. aureus strains.

\section{The distribution of mecC-MRSA in hedgehogs}

We first examined the geographical distribution and population structure of mecC-MRSA in European hedgehogs, which inhabit large parts of Europe as a result of postglacial expansion from Pleistocene refugia $^{20}$. European hedgehogs have also become widespread in New Zealand after a series of introductions from the UK between 1869 and 1892 (ref. ${ }^{21}$ ). We analysed 828 samples from the nasal area, skin and feet of 276 hedgehogs originating from 16 wildlife rescue centres in 10 European countries and 2 wildlife rescue centres in New Zealand (Fig. 1 and Extended Data Fig. 1). mecC-MRSA was present in 101 of the 172 hedgehogs ( 222 out of 516 samples) from England and Wales $(66 \%, 81$ out of 123$)$, Czech Republic $(50 \%, 6$ out of 12$)$, Denmark (50\%, 11 out of 22$)$, Portugal $(29 \%, 2$ out of 7$)$ and New Zealand $(6 \%, 1$ out of 17), therefore extending the known geographical distribution of mecC-MRSA in hedgehogs (Fig. 1 and Extended Data Fig. 1). By contrast, all 104 hedgehogs (312 samples) from Greece, Romania, Italy, France and Spain tested negative for mecC-MRSA. Whole-genome sequencing showed that the $222 \mathrm{mecC}$-MRSA isolates belonged to 6 clonal complexes, CC130 (75\%), CC1943 (15\%), CC2616 (6\%), CC425 (3\%), CC49 (1\%) and CC599 (1\%), of which CC130 had the most widespread distribution across western and central Europe (Fig. 1 and Extended Data Fig. 1). We screened all of the MRSA-negative hedgehog samples from our study $(n=606)$ for the presence of methicillin-susceptible S. aureus (MSSA) isolates belonging to the same clonal complexes as the mecC-MRSA isolates (Extended Data Fig. 1). This led to the identification of 22 MSSA isolates, including 13 CC49 isolates from Spain $(n=9)$, Denmark $(n=3)$ and Portugal $(n=1)$, and 9 CC130 isolates from England $(n=8)$ and Spain $(n=1)$.

The mecC gene encoding PBP2c is located immediately upstream of a blaZ gene (hereafter, bla $Z_{\mathrm{LGA} 251}$ ) on a chromosomally integrated mobile genetic element known as a type XI staphylococcal cassette chromosome mec (SCCmec). PBP2c and the bla $_{\mathrm{LGA} 251}$-encoded penicillinase are orthologues of the PBP2a enzyme and penicillinase produced by other $S$. aureus clones, although they share only $63 \%$ and $65 \%$ amino acid identities with each other, respectively ${ }^{10}$. Penicillinases have a narrower spectrum than $\mathrm{PBP} 2 \mathrm{a}$ and $\mathrm{PBP} 2 \mathrm{c}$ and provide resistance only to penicillin $G$ and other penicillinase-labile subclasses of penicillin. As expected, bla $Z_{\mathrm{LGA} 251}$ was present in the 222 mecC-MRSA isolates but absent in the 22 MSSA isolates. However, 14 of the MSSA isolates carried the blaZ gene found in other S. aureus clones (Supplementary Table 1).

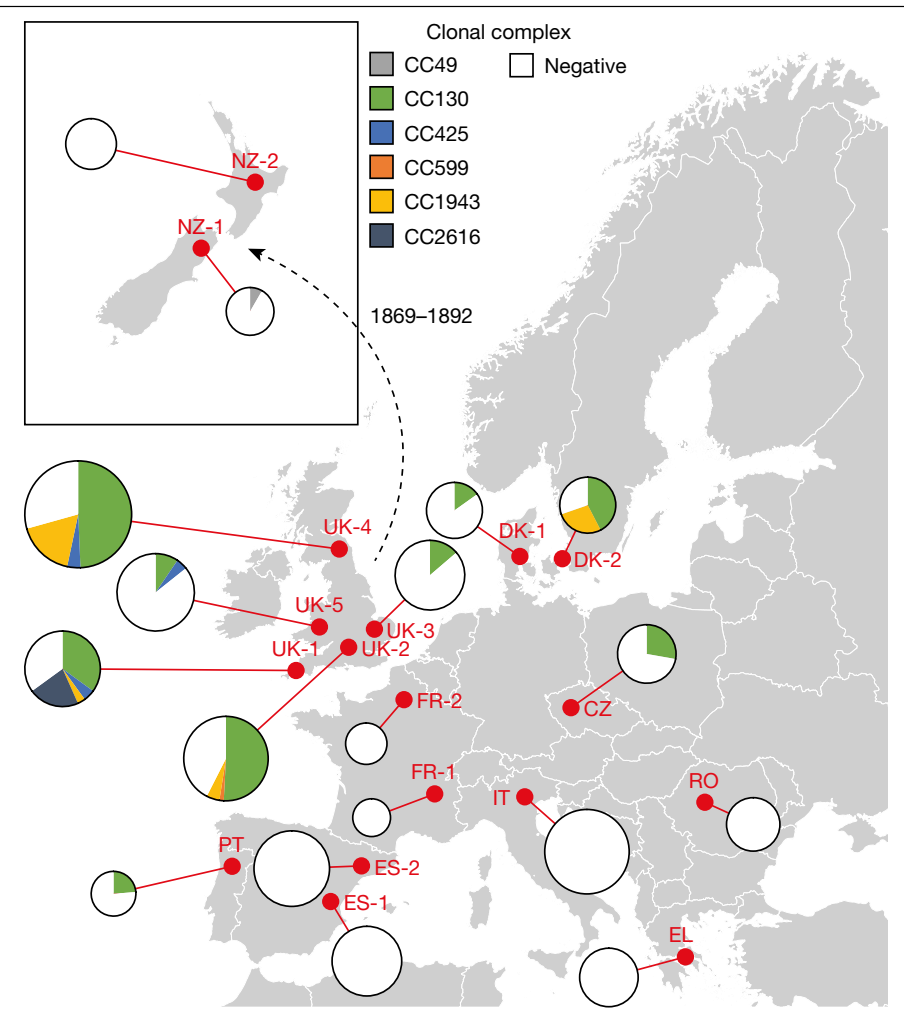

Fig. 1 | Distribution of mecC-MRSA clones in European and New Zealand hedgehog samples. The analysis included 828 samples from the nasal area, skin and feet of 276 hedgehogs originating from 16 wildlife rescue centres in 10 European countries and 2 wildlife rescue centres in New Zealand. The red dots indicate the sampling locations. The pie charts are connected to the sampling locations by a red line. The area of the pie chart is proportional to the number of samples from that location. The introduction of European hedgehogs into New Zealand from the UK between 1869 and 1892 is shown. A detailed description of the results is provided in Extended Data Fig. 1. Maps were provided by Eurostat under a Creative Commons Attribution 4.0 International (CC BY 4.0) licence; the administrative boundaries are copyright of EuroGeographics.

\section{Production of $\beta$-lactams by T. erinacei}

The abundance of mecC-MRSA in hedgehogs led us to speculate that antibiotic production by T.erinacei provides a selective environment in which mecC-MRSA isolates have an advantage over susceptible isolates. Genome sequencing and analysis of the T.erinacei type strain IMI 101051 (ATCC 28443) identified orthologues of $p c b A B, p c b C$ and pen $D E$, which are responsible for key steps in penicillin $\mathrm{G}$ production by Penicillium chrysogenum, as well as the Acremonium chrysogenum early cephalosporin $C$ biosynthetic genes cefD1 and cefD2, which are involved in the conversion of isopenicillin $\mathrm{N}$ into penicillin N (Fig. 2 and Extended Data Table1). By contrast, T. erinace i IMI 101051 lacked the $A$. chrysogenum late cephalosporin C biosynthetic genes cefEF and cefG. $P$. chrysogenum also carries cefD1 and cefD2 but is nevertheless incapable of producing cephalosporins due to the lack of $c e f E F$ and $c e f G^{22,23}$.

We processed four distinct culture broths of T.erinacei IMI 101051 for metabolic profiling using liquid chromatography-mass spectrometry (LC-MS) and molecular networking analysis. This led to the identification of two $\beta$-lactams, penicillin $G$ and 6-(5-hydroxy- $n$-valeramido)-pe nicillanic acid (KPN), both of which belong to the penicillin class of antibiotics (Extended Data Figs. 2 and 3 and Supplementary Fig. 1). KPN has to date been found only in culture broths of fungal strains belonging to the genus Paecilomyces ${ }^{24}$ and differs from penicillin $\mathrm{G}$ by having a unique side chain (Extended Data Figs. 2 and 3 and Supplementary Fig. 1). The biosynthetic pathway of KPN is currently unknown. 


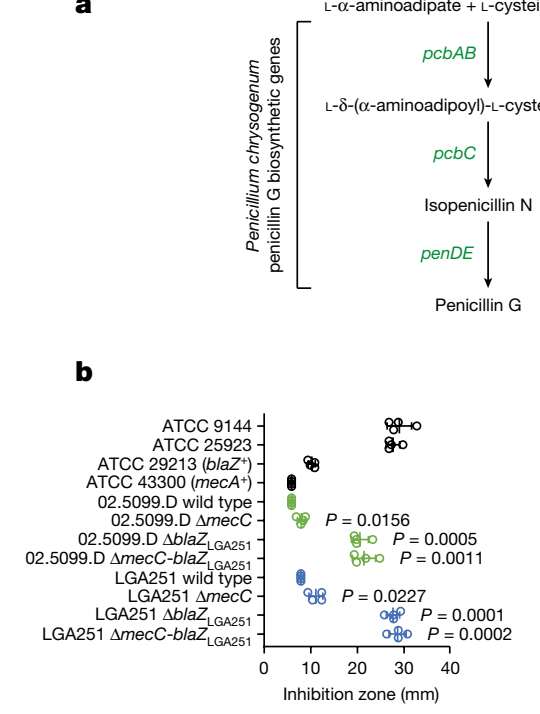

$L-\alpha$-aminoadipate $+L$-cysteine $+L$-valine

Fig. 2 Penicillin biosynthetic genes and antibiotic activity of $T$.erinacei IMI 101051.a, Schematic of the key steps in the biosynthesis of penicillin $G$ and cephalosporin C. The presence (green) or absence (red) of T.erinacei penicillin $\mathrm{G}$ and cephalosporin $\mathrm{C}$ biosynthetic genes is indicated. b, T. erinacei inhibition zones against a collection of $S$. aureus control strains (black) and two mecC-MRSA wild-type strains belonging to $\mathrm{CC} 130$ (green) and $\mathrm{CC} 425$ (blue) and their isogenic mutants. Two-tailed paired Student's $t$-tests were used to compare inhibition zones of each mutant to the corresponding wild-type strain. Data are mean \pm s.d.; $n=4$ biologically independent fungal culture extracts. A detailed description of the results is provided in Extended Data Fig. 4.

\section{Selection of mecC-MRSA by T. erinacei}

Four culture broths of T. erinacei IMI 101051 were screened for antibiotic activity against a collection of $S$. aureus control strains. All of the culture broths produced large inhibition zones against two penicillin-susceptible S. aureus strains-ATCC 9144 (Oxford S. aureus) and ATCC 25923-but much smaller zones against the penicillinase-producing S. aureus strain ATCC 29213 and the mec $A$-positive $S$. aureus strain ATCC 43300 (Fig. 2 and Extended Data Fig. 4). The role of mecC and $b l a Z_{\mathrm{LGA} 251}$ was assessed by screening the culture broths for antibiotic activity against two mecC-MRSA wild-type strains belonging to CC130 (02.5099.D) and CC425 (LGA251) and their isogenic mutants. The mutants with deleted mecC $(\Delta \mathrm{mecC})$, bla $_{\mathrm{LGA} 251}\left(\Delta\right.$ bla $\left._{\mathrm{LGA} 251}\right)$, and mecC and bla $_{\mathrm{LGA251}}\left(\Delta\right.$ mecC-bla $\left.Z_{\mathrm{LGA} 251}\right)$ produced significantly larger inhibition zones compared with the corresponding wild-type strains, although the zones of the $\Delta b l a Z_{\mathrm{LGA251}}$ and $\Delta$ mecC-bla $Z_{\mathrm{LGA} 251}$ mutants were larger compared with the zones of the $\Delta m e c C$ mutants (Fig. 2 and Extended Data Fig. 4). These results indicate that $m e c C$ and $b l a Z_{\mathrm{LGA} 251}$ both contribute to the reduced susceptibility of mecC-MRSA to penicillin $\mathrm{G}$ and KPN present in culture broths of T. erinacei IMI 101051.

\section{Evolutionary history of mecC-MRSA}

We sought to infer the evolutionary histories of S. aureus CC130, CC425 and CC1943, which constitute the most successful mecC-MRSA clones in Europe $^{10,11,25}$. For this purpose, we collected and sequenced 786 mecC-MRSA and 127 MSSA CC130, CC425 and CC1943 isolates selected to represent the known geographical distribution (mainly western and central Europe) and host repertoire (mainly humans, cattle, sheep, goats and wild animals) of each clone (Supplementary Table1). We used core-genome single-nucleotide polymorphism (SNP) diversity and isolation dates to infer time-scaled phylogenies of these isolates and the 205 mecC-MRSA and 9 MSSA CC130, CC425 and CC1943 isolates collected from hedgehogs (Supplementary Table 1). The sequencing data were processed for pan-genome analysis to identify antibiotic-resistance genes (ARGs) and mobile genetic elements that encode human- and ruminant-specific immune modulators that are involved in host switching events, including a phage-encoded immune evasion cluster-1(IEC-1) enabling $S$. aureus to evade the human innate immune response and a staphylococcal pathogenicity island (SaPI)-encoded $v w b$ gene ( $\left.v w b_{\mathrm{SaP}}\right)$, which encodes a von Willebrand factor-binding protein with coagulase activity against ruminant plasma ${ }^{26}$.

We also sought to infer a time-scaled phylogeny of the 991 type XI SCCmec elements containing the mecC and bla $Z_{\mathrm{LGA} 251}$ genes but the correlation between root-to-tip distances and isolation dates was too weak with a coefficient of determination $R^{2}=-0.05$ (Extended Data Fig. 5). Instead, we used the topology of the type XISCCmec phylogeny to identify monophyletic mecC-MRSA lineages harbouring orthologous type XI SCCmec elements. The type XI SCCmec elements could be traced back to seven nodes that were connected to each other on a long backbone. Each of the backbone nodes and its orthologous descendants received the same letter designation to reflect their genetic relationship (A to G) (Fig. 3 and Supplementary Fig. 2). Manual mapping of the tips onto the CC130, CC425 and CC1943 phylogenies, and vice versa, enabled us to assign the mecC-MRSA isolates to 16 monophyletic lineages harbouring orthologous type XI SCCmec elements (Fig. 3 and Supplementary Figs. 2-5). The 129 mecC-MRSA CC1943 isolates could be divided into three lineages (C1 to C3), which probably originated in the early-to-late 1800 s, long before the first $\beta$-lactam-penicillin $\mathrm{G}$-became widely available as a therapeutic option in the 1940s (Fig. 3). The 786 mecC-MRSA CC130 isolates and 76 mecC-MRSA CC425 isolates belonged to 10 and 3 lineages (A1 to A10 and B1 to B3, respectively) (Fig. 3). Several of these lineages also originated in the pre-antibiotic era (Fig. 3). Most mecC-MRSA isolates lacked $v w b_{\mathrm{SaPI}}(96 \%, 949$ out of 991$)$ and IEC-1 $(100 \%, 990$ out of 991) and were genotypically susceptible to non- $\beta$-lactam antibiotics (Supplementary Figs. 3-5). The largest mecC-MRSA CC425 lineage (CC425:B3) had a unique evolutionary trajectory with signs of adaptation to ruminants (Supplementary Fig. 4). The basal mecC-MRSA CC425:B3 isolates probably originated in England during the early 1940s and shared epidemiological and genetic characteristics with the other mecC-MRSA lineages: they were associated with multiple hosts, including hedgehogs, cattle and humans, and lacked $v w b_{\mathrm{SaPl}}$ and IEC-1. By contrast, their descendants (CC425:B3.1) harboured $v w b_{\mathrm{SaP}}$, were restricted to cattle and humans in southwest England and probably diverged during the 1960s (date of the most recent common ancestor (MRCA), 1965; 95\% confidence interval (CI), 1926-1986). Our analysis revealed that some of the mecC-MRSA lineages (CC130:A2, CC425:B3 and CC1943:C1) carried unique variants of the type XISCCmec element, supporting that they have evolved through vertical inheritance from the MRCA of each mecC-MRSA lineage, whereas others shared the same type XI SCCmec variant (Fig. 3 and Supplementary Figs. 2-5). The latter findings could be the result of either purifying (negative) selection, convergent evolution, homologous recombination between different mecC-MRSA lineages or horizontal gene transfer.

To better understand the potential role of horizontal gene transfer in the evolution of the three early mecC-MRSA CC1943 lineages, we determined the smallest number of sublineages that were present at a given time point, which is also the smallest number of acquisition events that could explain the presence of the same type XI SCCmec variant in all sublineages (Extended Data Fig. 6). The analysis showed that mecC-MRSA CC1943 consisted of 15 sublineages in 1940, just before penicillin $\mathrm{G}$ became available as a therapeutic option. It has previously been estimated that SCCmec was acquired in a single horizontal gene transfer event in three of the major hospital- and community-associated MRSA clones ${ }^{4,27,28}$, although multiple introductions have also been reported ${ }^{29}$. Thus, it is more plausible to assume that the type XI SCCmec element was present in the MRCA of each 


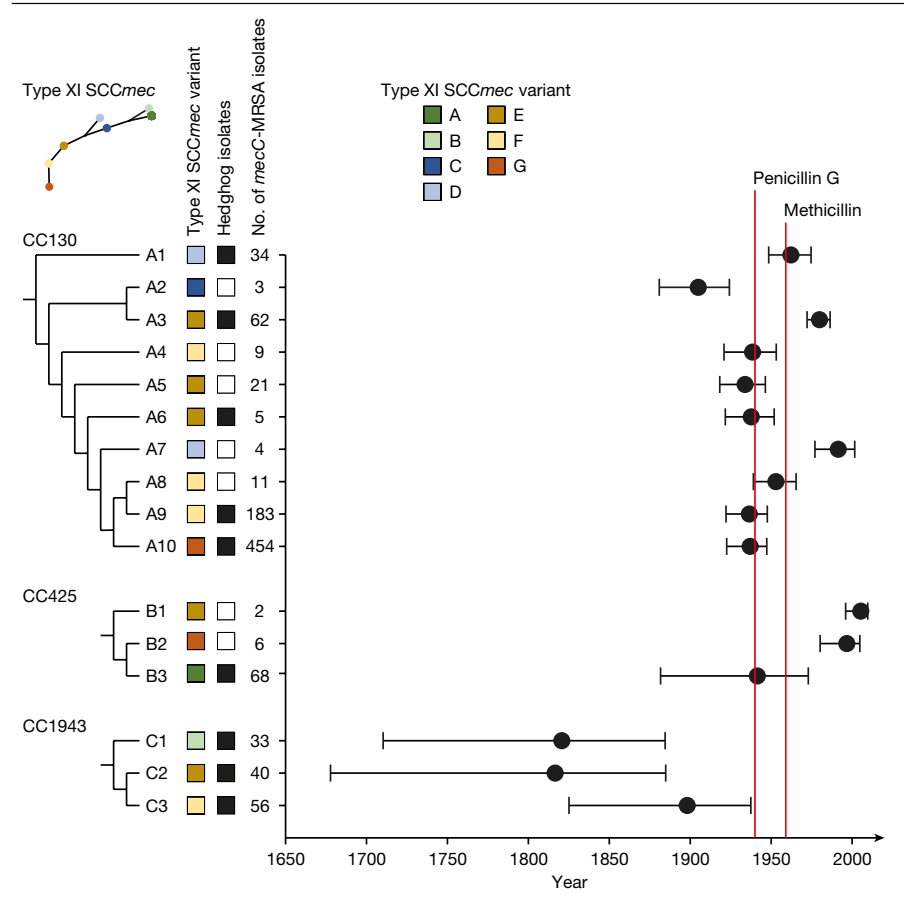

Fig. 3 | Timeline of mecC-MRSA CC130, CC425 and CC1943 evolution in Europe. Manual mapping of the tips on the type XISCCmec phylogeny onto the CC130, CC425 and CC1943 phylogenies, and vice versa, enabled us to assign the mecC-MRSA isolates to 16 monophyletic lineages containing orthologous type XISCCmec elements $(\mathrm{A}-\mathrm{G})$. The trees are redrawn from Supplementary Figs. 2-5 to illustrate the branching order of the different type $\mathrm{XISCC} m e c$ variants and $m e c C$-MRSA lineages. Branch lengths are not drawn to scale. The presence and absence of hedgehog isolates in a given lineage are shown as black and white boxes, respectively. A detailed description of the geographical distribution and host range of major mecC-MRSA CC130, CC425 and CC1943 lineages is provided in Extended Data Fig. 7. The estimated date of the most recent common ancestor and $95 \%$ confidence interval of each mecC-MRSA lineage are illustrated by filled circles and horizontal lines, respectively. The introduction of penicillin $\mathrm{G}$ and methicillin as therapeutic options is indicated by red lines.

mecC-MRSA CC1943 lineage rather than assuming horizontal gene transfer into each of the 15 sublineages within a few years at the beginning of the antibiotic era. Vertical inheritance of the type XI SCCmec element is also consistent with the apparent absence of admixture between the different mecC-MRSA CC1943 lineages despite the fact that they are often found in hedgehogs within the same geographical area (Extended Data Fig. 7). Notably, the type XISCCmec variants found in mecC-MRSA CC1943:C2 (E) and CC1943:C3 (F) were each other's neighbours on the type XISCCmec phylogeny, and it is therefore possible that the type XI SCCmec element was acquired even earlier (date of the MRCA, 1737; 95\% CI, 1562-1824).

MSSA isolates comprised $8 \%$ ( 67 out of 851 ), $47 \%$ ( 68 out of 144 ) and $0.8 \%$ (1 out of 130) of the CC130, CC425 and CC1943 isolates, respectively. The vast majority of the MSSA CC130 and MSSA CC 425 isolates, but not the single MSSA CC1943 isolate, were basal to the corresponding $m e c C$-MRSA lineages (Supplementary Figs. 3-5). Most of the basal MSSA CC130 isolates originated from sheep and goats in Italy, France, Spain and Norway and harboured $v w b_{\text {SaPl }}$. Moreover, some of the isolates carried ARGs against antibiotics that are used to treat infections in sheep and goats, including the two tetracycline-resistance genes tet $(\mathrm{K})$ and tet $(\mathrm{L})$, the blaZ gene found in other $S$. aureus clones, the chloramphenicol resistance gene cat and the macrolide resistance gene erm (C). The earliest branching CC425 lineages were epidemiologically and genetically diverse with respect to host range and the presence/absence of the type XI SCCmec element, $v w b_{\mathrm{SaPI}}$ and ARGs, although most originated from wild animals in Spain and lacked the aforementioned genetic determinants. Together, these findings suggest that $\mathrm{CC} 130$ and $\mathrm{CC} 425$ emerged from distinct ruminant and wildlife reservoirs in Europe and that methicillin resistance is an acquired phenotype within these clones.

\section{Population dynamics of mecC-MRSA}

Hedgehogs constitute a large reservoir of mecC-MRSA clones, as demonstrated here and elsewhere ${ }^{8,9}$, whereas mecC-MRSA isolates are present at much lower frequencies in humans, domesticated animals and other wild animals. Hedgehog isolates were present in 9 out of the 16 mecC-MRSA lineages, including the 8 largest ( $\geq 25$ isolates) and 3 earliest (200-130 years ago) lineages (Fig. 3). The 2 largest mecC-MRSA CC130 lineages (CC130:A9 and CC130:A10) encompassed 67\% (232 out of 344), 65\% (339 out of 520) and 43\% (66 out of 153) of all mecC-MRSA isolates from hedgehogs, humans and other sources, respectively, and had the broadest geographical ranges in western and central Europe (Extended Data Fig. 7 and Supplementary Figs. 3-5). Several of the major mecC-MRSA CC130 and CC1943 lineages (such as CC130:A9, CC130:A10, CC1943:C1, CC1943:C2 and CC1943:C3) contained isolates that were separated by wide expanses of seawater, reflecting numerous long-distance dispersal events between British and Danish islands and mainland Europe within the past 200 years (Extended Data Fig. 7 and Supplementary Figs. 3-5). By contrast, analysis of the fine-scale population structure of mecC-MRSA isolates from hedgehogs revealed a detailed pattern of diversifications over short distances, resulting in substantial concordance between genetic clusters and geography at the local level (Supplementary Figs. 3-5). The observed clustering is consistent with the limited dispersal capacity of hedgehogs and the effects of habitat fragmentation.

Assuming that hedgehogs act as local reservoirs (sources) of mecC-MRSA in Denmark, theoretical considerations predict that secondary transmission to other hosts (sinks) would lead to similar patterns of genetic variation within these secondary hosts cohabiting the same geographical region. To examine this theory, we determined the local population structures of two broad collections of Danish mecC-MRSA isolates from hedgehogs and humans covering the geographical ranges of two of the three Danish hedgehog subpopulations, namely Jutland (a peninsula connected to continental Europe) and major islands $\mathrm{s}^{30}$. Hedgehog and human isolates from the geographical range of the remaining hedgehog subpopulation (the small island of Bornholm) were excluded from the analysis due to their small sample size (nine isolates). Most of the CC130:A10 isolates from Jutland formed a distinct sublineage (CC130:A10.1) together with isolates from other parts of Europe (Supplementary Fig. 3). As a consequence, CC130:A10.1 isolates were treated as a separate group in the analysis. The results revealed distinct patterns of regional dispersal with little overlap between Jutland and the major islands and a notable correlation between the population structures of hedgehog and human isolates at the regional level ( $P=0.0149$, two-sided Wilcoxon matched-pairs signed-rank test) (Fig. 4 and Extended Data Fig. 8). These findings support the hypothesis that human isolates originate from local hedgehog reservoirs, although it should be noted that the data presented here do not provide evidence for directionality or rule out the involvement of other animal reservoirs (such as livestock) as part of the transmission chains.

\section{Discussion}

This research shows that hedgehogs are a natural reservoir of zoonotic mecC-MRSA lineages that predate the antibiotic era, which is inconsistent with the commonly accepted view that widespread resistance in clinical pathogens is a modern phenomenon that is driven by our use of antibiotics in human and veterinary medicine.

Data on the prevalence of mecC-MRSA in humans and different animal species indicate that hedgehogs are the most likely primary host in 


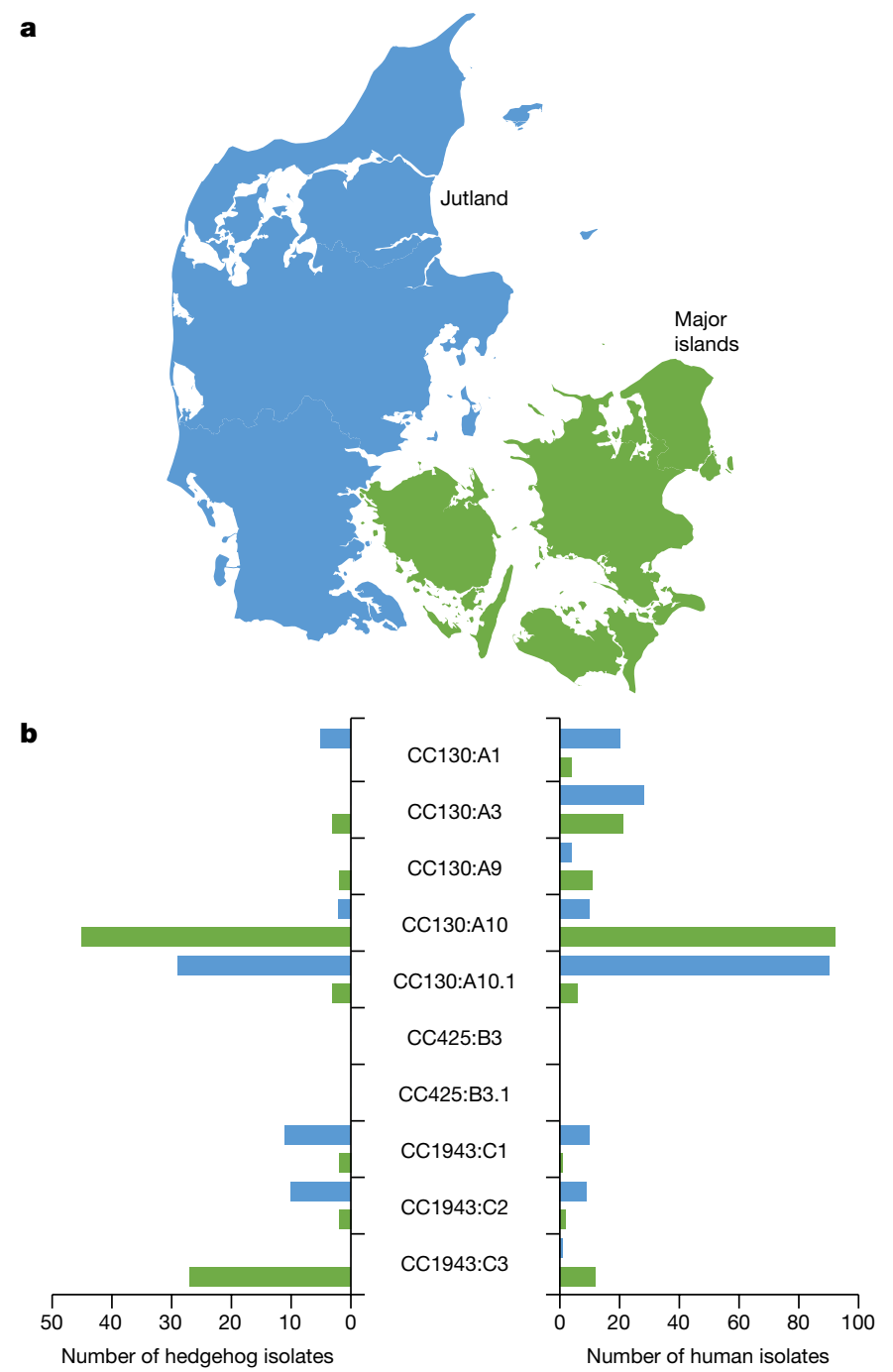

Fig. 4 Population structures of Danish mecC-MRSA isolates from hedgehogs and humans. a, The map of Denmark shows the geographical ranges of two of the three hedgehog subpopulations in Jutland and on the major islands. b. The geographical distribution of major mecC-MRSA CC130, CC425 and CC1943 lineages in two broad collections of mecC-MRSA isolates recovered from hedgehogs $(n=141)$ and humans $(n=327)$ in Jutland and on the major islands. Hedgehog and human isolates from the remaining hedgehog subpopulation on the small island of Bornholm (not shown) were excluded from the analysis due to their small sample size $(n=9)$. A detailed map of the sampling locations is provided in Extended Data Fig. 8. Maps were provided by Eurostat under a Creative Commons Attribution 4.0 International (CC BY 4.0) licence; the administrative boundaries are copyright of EuroGeographics.

some countries. For example, in Denmark, the prevalence in hedgehogs is considerably higher than in cattle (veal calves and bulk tank milk), sheep and goats $(61 \% \text { versus } 0.0-1.1 \%)^{8,31,32}$, and the number of human cases is relatively low (3-36 cases per year) ${ }^{25}$. This is further supported by our finding that $\mathrm{mecC}$-MRSA generally lacks genetic markers of human and ruminant adaptation, with the notable exception of the CC425:B3.1 lineage that has undergone a host jump from hedgehogs to cattle in southwest England. Before this study, dairy cows were considered to be the most likely reservoir of $m e c C$-MRSA and a major source of zoonotic infections in humans. This hypothesis was supported by the fact that $\beta$-lactams are commonly used to treat bovine mastitis, as shown by sales data from Denmark and the $\mathrm{UK}^{33,34}$. However, our findings strongly suggest that most mecC-MRSA lineages originate from hedgehogs, although dairy cows and other domesticated animals probably act as intermediate hosts and vectors in zoonotic transmission from hedgehogs to humans, as previously demonstrated ${ }^{35}$.

Here we show that the $T$. erinacei type strain IMI 101051 from New Zealand produces two $\beta$-lactams-penicillin $\mathrm{G}$ and KPN-and that mecC and bla $Z_{\mathrm{LGA251}}$ both contribute to reduced susceptibility of mecC-MRSA to these antibiotics. Previous studies have established that T. erinacei is widespread among hedgehogs in New Zealand and northwestern Europe and that isolates from both continents produce a penicillinase-labile penicillin-like substance ${ }^{12-19}$, and a recent study characterized penicillin G from $T$. erinacei in Sweden (the presence of other $\beta$-lactams was not investigated ${ }^{19}$. This suggests that penicillin-producing T.erinacei isolates were circulating in European hedgehogs long before they were introduced into New Zealand in the late 1800s and that methicillin resistance first emerged in Europe as a co-evolutionary adaptation of $S$. aureus to colonization of hedgehogs. By contrast, it cannot be ruled out that clinical use of antibiotics in humans and livestock has contributed to the evolution of some of the younger mecC-MRSA CC130 and CC425 lineages, although only one of these lineages (CC425:B3) showed signs of adaptation to either of these hosts. Our findings indicate that seven of the younger mecC-MRSA lineages (CC130:A3, CC130:A4, CC130:A5, CC130:A6, CC130:A8, CC130:A9 and CC425:B1) have acquired their type XI SCCmec variants from the early mecC-MRSA CC1943:C2 and CC1943:C3 lineages (Fig. 3 and Supplementary Figs. 2-5). The type XISCCmec element has also been found at low frequencies in coagulase-negative Staphylococcus species from wild animals and livestock but the evolutionary links between these potential donors and mecC-MRSA remain to be investigated ${ }^{36}$.

Our analyses suggest that most mecC-MRSA transmission events within hedgehog populations and between hedgehogs and secondary hosts are highly localized. The finding that some human mecC-MRSA isolates probably originate from local hedgehog reservoirs indicates that mecC-MRSA has been a cause of sporadic infections in humans for the past 200 years, more than a century before MRSA was first identified in patients in 1960 (ref. $^{3}$ ). The host interactions that lead to zoonotic transmission probably include direct contact with hedgehogs or contact with secondary animal hosts such as dairy cows, as previously shown for $T$. erinacei (the cause of 'hedgehog ringworm' in humans) ${ }^{13}$. We also identified several long-distance dispersal events between British and Danish islands and mainland Europe. The connections that bridge geographically isolated hedgehog populations are poorly understood but might involve oversea movements of humans and livestock. Furthermore, a recent report of mecC-MRSA in white storks raises the possibility that migratory birds could be efficient long-distance carriers ${ }^{37}$.

$\beta$-Lactams target PBPs that catalyse carboxypeptidase and transpeptidase reactions during bacterial cell wall synthesis, whereby they inhibit cross-linking of neighbouring peptidoglycan strands. The primary mechanisms of $\beta$-lactam resistance in $S$. aureus are enzymatic cleavage of the amide bond in the $\beta$-lactam ring of penicillinase-labile penicillins by blaZ-encoded penicillinases and the production of $m e c A$ or mecC-encoded PBP2a and PBP2c, respectively, with a decreased affinity for a broad spectrum of $\beta$-lactams. Our findings support that both mechanisms contribute to protection against penicillin production by T. erinacei in hedgehogs, although it should be noted that the $\Delta m e c C$ mutants produced smaller inhibition zones than the $\Delta b l a Z_{\mathrm{LGA} 251}$ mutants. This might be due to the fact that $P B P 2 c$ has a relatively high binding affinity for penicillins compared with $\mathrm{PBP} 2 \mathrm{a}^{38}$. It is also possible that PBP2c provides additional ecological benefits, such as protection against cephalosporin-producing fungi and bacteria that occur naturally in all environments ${ }^{39}$.

mecC and $b l a Z_{\mathrm{LGA} 251}$ have also been found on a pseudo-SCCmec element ( $\left.\Psi \mathrm{SCC}_{\mathrm{Cec}} \mathrm{P}_{\mathrm{P} 085}\right)$ in Staphylococcus edaphicus, a soil-dwelling bacterial species isolated from Antarctica ${ }^{40}$. In contrast to the type XI SCCmec element, $\Psi \mathrm{SCC} \mathrm{mec}_{\mathrm{P} 5085}$ lacks the cassette chromosome recombinase (ccr) genes that are responsible for movement (excision and integration) of SCC $\mathrm{mec}^{40}$. Several studies have identified ARGs in 
ancient and modern samples and bacteria from natural environment such as soil ${ }^{41-43}$. Yet, the environmental resistome shows limited potential for horizontal gene transfer and, for this reason, the contribution of environmental ARGs to resistance in human pathogens has so far been controversial $^{44}$. It seems reasonable to assume that the microbiota of wild animals have greater exposure to the environmental resistome than the human microbiota and are therefore more likely to acquire environmental ARGs. Thus, wild animals might represent a hitherto unrecognized conduit through which environmental ARGs can be transferred to clinical pathogens.

We acknowledge some limitations of our study. Although we broadly sampled hedgehogs across Europe and New Zealand, our study represents only a small part of the geographical range and a small number of hedgehog samples in most countries. Thus, the distribution and diversity of mecC-MRSA in Europe and New Zealand might be larger than documented here. We cannot account for the potential effect of transmission within the different wildlife rescue centres. To better understand the transmission dynamics, we examined the frequency of potential transmission events of mecC-MRSA CC130 within the different facilities using a range of maximum pairwise SNP distance thresholds to define a cluster (Extended Data Fig. 9). Using a conservative cut-off of 25 SNPs (transmission age $<6$ months $)^{45}, 25 \%$ ( 683 out of 2,783 ) of the mecC-MRSA CC130 isolate pairs collected within the same facility belonged to a potential transmission cluster. It is therefore possible that the prevalence of mecC-MRSA in hedgehogs kept in such facilities is higher than in the wild, although it is also probable that some of these potential transmission events occurred in the wild before admission to the facility. Notably, a previous study found that there is only a slightly lower prevalence of mecC-MRSA in hedgehogs that died in the wild compared with hedgehogs that died while staying in a wildlife rescue centre $^{8}$, which is consistent with our finding that most hedgehogs acquired mecC-MRSA outside the facility. We were unable to test the hedgehogs for carriage of $T$. erinacei, because ethical constraints precluded us from collecting appropriate tissues (skin scrapings, hair and spines) for fungal culture, which leaves some important questions unanswered. For example, it remains unclear whether penicillin-producing $T$. erinace $i$ isolates are present throughout Europe and whether there is a link between their distribution and the geographical range of $m e c C$-MRSA.

In conclusion, we describe the ecological and evolutionary mechanisms that led to the emergence of methicillin resistance in the pre-antibiotic era, possibly as a co-evolutionary adaptation of $S$. aureus to colonization of dermatophyte-infected hedgehogs. These results underscore the importance of taking a broad One Health perspective on antibiotic resistance that recognises the role of natural selection in wild animals and the connectivity of natural, agricultural and human ecosystems in the evolution and spread of antibiotic-resistant pathogens.

\section{Online content}

Any methods, additional references, Nature Research reporting summaries, source data, extended data, supplementary information, acknowledgements, peer review information; details of author contributions and competing interests; and statements of data and code availability are available at https://doi.org/10.1038/s41586-021-04265-w.

1. Davies, J. \& Davies, D. Origins and evolution of antibiotic resistance. Microbiol. Mol. Biol. Rev. 74, 417-433 (2010).

2. European Centre for Disease Prevention and Control, European Medicines Agencies. The Bacterial Challenge: Time to React. A Call to Narrow the Gap Between Multidrug-Resistant Bacteria in the EU and the Development of New Antibacterial Agents https://ecdc.europa. eu/sites/portal/files/media/en/publications/Publications/0909_TER_The_Bacterial_ Challenge_Time_to_React.pdf (2009).

3. Jevons, M. P. "Celbenin"-resistant Staphylococci. Br. Med. J. 1, 124-125 (1961).

4. Harkins, C. P. et al. Methicillin-resistant Staphylococcus aureus emerged long before the introduction of methicillin into clinical practice. Genome Biol. 18, 130 (2017).
5. Chambers, H. F. \& DeLeo, F. R. Waves of resistance: Staphylococcus aureus in the antibiotic era. Nat. Rev. Microbiol. 7, 629-641 (2009).

6. Price, L. B. et al. Staphylococcus aureus CC398: host adaptation and emergence of methicillin resistance in livestock. mBio 3, e00305-11 (2012).

7. Global Priority List of Antibiotic-Resistant Bacteria to Guide Research, Discovery, and Development of New Antibiotics http://www.who.int/medicines/publications/ WHO-PPL-Short_Summary_25Feb-ET_NM_WHO.pdf?ua=1 (WHO, 2017).

8. Rasmussen, S. L. et al. European hedgehogs (Erinaceus europaeus) as a natural reservoir of methicillin-resistant Staphylococcus aureus carrying mecC in Denmark. PLOS ONE 14, e0222031 (2019)

9. Bengtsson, B. et al. High occurrence of mecC-MRSA in wild hedgehogs (Erinaceus europaeus) in Sweden. Vet. Microbiol. 207, 103-107 (2017).

10. García-Álvarez, L. et al. Methicillin-resistant Staphylococcus aureus with a novel mecA homologue in human and bovine populations in the UK and Denmark: a descriptive study. Lancet Infect. Dis. 11, 595-603 (2011).

11. Paterson, G. K., Harrison, E. M. \& Holmes, M. A. The emergence of mecC methicillin-resistant Staphylococcus aureus. Trends Microbiol. 22, 42-47 (2014).

12. Marples, M. J. \& Smith, J. M. B. The hedgehog as a source of human ringworm. Nature 188 867-868 (1960)

13. English, M. P., Evans, C. D., Hewitt, M. \& Warin, R. P. “Hedgehog ringworm”. Br. Med. J. 1, 149-151 (1962).

14. Smith, J. M. B. \& Marples, M. J. A natural reservoir of penicillin-resistant strains of Staphylococcus aureus. Nature 201, 844 (1964).

15. Smith, J. M. B. \& Marples, M. J. Dermatophyte lesions in the hedgehog as a reservoir of penicillin-resistant staphylococci. J. Hyg. 63, 293-303 (1965).

16. Smith, J. M. B. Staphylococcus aureus strains associated with the hedgehog Erinaceus europaeus. J. Hyg. Camb. 63, 293-303 (1965).

17. Morris, P. \& English, M. P. Trichophyton mentagrophytes var. erinacei in British hedgehogs. Sabouraudia 7, 122-128 (1969).

18. Le Barzic, C. et al. Detection and control of dermatophytosis in wild European hedgehogs (Erinaceus europaeus) admitted to a French wild life rehabilitation centre. J. Fungi 7, 74 (2021).

19. Dube, F., Söderlund, R., Salomonsson, M. L., Troell, K. \& Börjesson, S. Benzylpenicillin-producing Trichophyton erinacei and methicillin resistant Staphylococcus aureus carrying the mecC gene on European hedgehogs: a pilot-study. BMC Microbiol. 21, 212 (2021).

20. Hewitt, G. The genetic legacy of the Quaternary ice ages. Nature 405, 907-913 (2000).

21. Brockie, R. E. Distribution and abundance of the hedgehog (Erinaceus europaeus) L. in New Zealand, 1869-1973. N. Z. J. Zool. 2, 445-462 (1975)

22. van den Berg, M. A. et al. Genome sequencing and analysis of the filamentous fungus Penicillium chrysogenum. Nat. Biotechnol. 26, 1161-1168 (2008).

23. Ullán, R. V., Campoy, S., Casqueiro, J., Fernández, F. J. \& Martín, J. F. Deacetylcephalosporin $\mathrm{C}$ production in Penicillium chrysogenum by expression of the isopenicillin $\mathrm{N}$ epimerization, ring expansion, and acetylation genes. Chem. Biol. 14, 329-339 (2007).

24. Kitano, K. et al. A novel penicillin produced by strains of the genus Paecilomyces. J. Ferment. Technol. 54, 705-711 (1976).

25. Petersen, A. et al. Epidemiology of methicillin-resistant Staphylococcus aureus carrying the novel mecC gene in Denmark corroborates a zoonotic reservoir with transmission to humans. Clin. Microbiol. Infect. 19, E16-E22 (2013).

26. Richardson, E. J. et al. Gene exchange drives the ecological success of a multi-host bacterial pathogen. Nat. Ecol. Evol. 2, 1468-1478 (2018).

27. Holden, M. T. G. et al. A genomic portrait of the emergence, evolution, and global spread of a methicillin-resistant Staphylococcus aureus pandemic. Genome Res. 23, 653-664 (2013).

28. Strauß, L. et al. Origin, evolution, and global transmission of community-acquired Staphylococcus aureus ST8. Proc. Natl Acad. Sci. USA 114, E10596-E10604 (2017).

29. Nübel, U. et al. Frequent emergence and limited geographic dispersal of methicillinresistant Staphylococcus aureus. Proc. Natl Acad. Sci. USA 105, 14130-14135 (2008).

30. Rasmussen, S. L., Nielsen, J. L., Jones, O. R., Berg, T. B. \& Pertoldi, C. Genetic structure of the European hedgehog (Erinaceus europaeus) in Denmark. PLoS ONE 15, e0227205 (2020).

31. Hansen, J. E. et al. LA-MRSA CC398 in dairy cattle and veal calf farms indicates spillover from pig production. Front. Microbiol. 10, 2733 (2019).

32. Eriksson, J. Espinosa-Gongora, C., Stamphøj, I., Larsen, A. R. \& Guardabassi, L. Carriage frequency, diversity and methicillin resistance of in Danish small ruminants. Vet. Microbiol. 163, 110-115 (2013)

33. Danish Integrated Antimicrobial Resistance Monitoring and Research Programme. DANMAP 2019: Use of Antimicrobial Agents and Occurrence of Antimicrobial Resistance in Bacteria From Food Animals, Food, and Humans in DENMARK https://www.danmap.org/-/ media/Sites/danmap/Downloads/Reports/2019/DANMAP_2019.ashx?la=da\&hash=AA193 9EB449203EF0684440AC1477FFCE2156BA5 (2020).

34. Veterinary Medicines Directorate. UK Veterinary Antibiotic Resistance and Sales Surveillance Report https://assets.publishing.service.gov.uk/government/uploads/system/ uploads/attachment_data/file/950126/UK-VARSS_2019_Report_2020-TPaccessible.pdf (2020).

35. Harrison, E. M. et al. Whole genome sequencing identifies zoonotic transmission of MRSA isolates with the novel mecA homologue mecC. EMBO Mol. Med. 5, 509-515 (2013).

36. Loncaric, I. et al. Characterization of $m e c C$ gene-carrying coagulase-negative Staphylococcus spp. isolated from various animals. Vet. Microbiol. 230, 138-144 (2019).

37. Gómez, P. et al. Detection of MRSA ST3061-t843-mecC and ST398-t011-mecA in white stork nestlings exposed to human residues. J. Antimicrob. Chemother. 71, 53-57 (2016).

38. Kim, C. et al. Properties of a novel PBP2A protein homolog from Staphylococcus aureus strain LGA251 and its contribution to the $\beta$-lactam-resistant phenotype. J. Biol. Chem. 287, 36854-36863 (2012)

39. Tahlan, K. \& Jensen, S. E. Origins of the $\beta$-lactam rings in natural products. J. Antibiot. 66, 401-419 (2013) 
40. Pantůček, R. et al. Staphylococcus edaphicus sp. nov. isolated in Antarctica harbors the mecC gene and genomic islands with a suspected role in adaptation to extreme environment. Appl. Environ. Microbiol. 84, e01746-17 (2018).

41. D'Costa, V. M., et al. Antibiotic resistance is ancient. Nature 477, 457-461 (2011).

42. Allen, H. K., Moe, L. A., Rodbumrer, J., Gaarder, A. \& Handelsman, J. Functional metagenomics reveals diverse beta-lactamases in a remote Alaskan soil. ISME J. 3, 243-251 (2009).

43. Forsberg, K. J. et al. The shared antibiotic resistome of soil bacteria and human pathogens. Science 337, 1107-1111 (2012).

44. Forsberg, K. J. et al. Bacterial phylogeny structures soil resistomes across habitats. Nature 509, 612-616 (2014)

45. Coll, F. et al. Definition of a genetic relatedness cutoff to exclude recent transmission of meticillin-resistant Staphylococcus aureus: a genomic epidemiology analysis. Lancet Microbe 1, e328-e335 (2020).

Publisher's note Springer Nature remains neutral with regard to jurisdictional claims in published maps and institutional affiliations.

Open Access This article is licensed under a Creative Commons Attribution 4.0 International License, which permits use, sharing, adaptation, distribution and reproduction in any medium or format, as long as you give appropriate credit to the original author(s) and the source, provide a link to the Creative Commons license and indicate if changes were made. The images or other third party material in this article are included in the article's Creative Commons license, unless indicated otherwise in a credit line to the material. If material is not included in the article's Creative Commons license and your intended use is not permitted by statutory regulation or exceeds the permitted use, you will need to obtain permission directly from the copyright holder. To view a copy of this license, visit http://creativecommons.org/licenses/by/4.0/.

\section{(C) The Author(s) 2022}

'Department of Bacteria, Parasites \& Fungi, Statens Serum Institut, Copenhagen, Denmark. ${ }^{2}$ Department of Veterinary Medicine, University of Cambridge, Cambridge, UK. ${ }^{3}$ Royal Botanic Gardens, Kew, Richmond, UK. ${ }^{4}$ Institute of Microbiology, University of Veterinary Medicine, Vienna, Austria. ${ }^{5}$ National Reference Center for Antimicrobial Resistance and Nosocomial Infections, Institute for Hygiene, Microbiology and Tropical Medicine, Ordensklinikum Linz Elisabethinen, Linz, Austria. ${ }^{6}$ National Reference Centre-Staphylococcus aureus, Department of Microbiology, Hôpital Erasme, Université libre de Bruxelles, Brussels, Belgium. ${ }^{7}$ Department of Animal Science and Food Processing, Faculty of Tropical AgriSciences, Czech University of Life Sciences Prague, Prague, Czech Republic. ${ }^{8}$ Department of Zoology, Charles University, Prague, Czech Republic. ${ }^{9}$ Department of Biology and Ecology, University of Ostrava, Ostrava, Czech Republic. ${ }^{10}$ European Programme for Public Health Microbiology Training (EUPHEM), European Centre for Disease Prevention and Control (ECDC), Stockholm, Sweden. "Infectious Disease Preparedness, Statens Serum Institut, Copenhagen, Denmark. ${ }^{12}$ Department of Chemistry and Bioscience, Aalborg University, Aalborg, Denmark. ${ }^{13}$ Wildlife Conservation Research Unit (WildCRU), Department of Zoology, University of Oxford, Tubney, UK. ${ }^{14}$ Department of Veterinary and Animal Sciences, Faculty of Health and Medical Sciences, University of Copenhagen, Frederiksberg, Denmark. ${ }^{15}$ National Food Institute, Technical University of
Denmark, Kongens Lyngby, Denmark. ${ }^{16}$ Expert Microbiology Unit, Department of Health Security, Finnish Institute for Health and Welfare, Helsinki, Finland. ${ }^{17}$ Microbiology Unit, Finnish Food Authority, Helsinki, Finland. ${ }^{18}$ Bacteriology Department and French National Reference Center for Staphylococci, Hospices Civils de Lyon, University of Lyon, Lyon, France. ${ }^{19}$ Friedrich Loeffler-Institute of Medical Microbiology, University Medicine Greifswald, Greifswald, Germany. ${ }^{20}$ Institute of Microbiology and Epizootics, Veterinary Faculty, Freie Universität Berlin, Berlin, Germany. ${ }^{21}$ Institute for Veterinary Food Science, Justus-Liebig University Giessen, Giessen, Germany. ${ }^{22}$ National Reference Centre for Staphylococci and Enterococci, Division Nosocomial Pathogens and Antibiotic Resistances, Department of Infectious Diseases, Robert Koch Institute, Wernigerode, Germany. ${ }^{23}$ Vet Med Labor GmbH, Kornwestheim, Germany. ${ }^{24}$ Dipartimento di Medicina Veterinaria, Università degli Studi di Milano, Lodi, Italy. ${ }^{25}$ Norwegian Veterinary Institute, Ås, Norway. ${ }^{26}$ Laboratory of Molecular Genetics, ITQB NOVA, Oeiras, Portugal. ${ }^{27}$ Laboratory of Microbiology and Infectious Diseases, The Rockefeller University, New York, NY, USA. ${ }^{28}$ Servicio de Microbiología, Hospital Universitario Lucus Augusti, Lugo, Spain. ${ }^{29}$ Department of Animal Health and Antimicrobial Strategies, National Veterinary Institute (SVA), Uppsala, Sweden. ${ }^{30}$ Department of Microbiology, Public Health Agency of Sweden, Solna, Sweden. ${ }^{31}$ Institute of Veterinary Bacteriology, University of Bern, Bern, Switzerland. ${ }^{32}$ Department of Bacteriology, Animal and Plant Health Agency, Weybridge, UK. ${ }^{33}$ Animal Health Trust, Newmarket, UK. ${ }^{34}$ Antimicrobial Resistance and Healthcare Associated Infections Reference Unit, UK Health Security Agency, London, UK. ${ }^{35}$ Clinical Microbiology and Public Health Laboratory, UK Health Security Agency, Addenbrooke's Hospital, Cambridge, UK. ${ }^{36}$ Department of Genetics, University of Cambridge, Cambridge, UK. ${ }^{37}$ Department of Medicine, University of Cambridge, Cambridge, UK. ${ }^{38}$ Institute of Infection and Immunity, St George's, University of London, London, UK. ${ }^{39}$ School of Medicine, University of St Andrews, St Andrews, UK. ${ }^{40}$ Scottish MRSA Reference Laboratory, NHS Greater Glasgow and Clyde, Stobhill Hospital, Glasgow, UK. ${ }^{41}$ SRUC Veterinary Services, Inverness, UK. ${ }^{42}$ The Royal (Dick) School of Veterinary Studies and Roslin Institute, University of Edinburgh, Easter Bush, UK. ${ }^{43}$ School of Life Sciences and Department of Statistics, University of Warwick, Warwick, UK. ${ }^{44}$ Wellcome Sanger Institute, Hinxton, UK. ${ }^{45}$ Department of Public Health and Primary Care, University of Cambridge, Cambridge, UK. ${ }^{46}$ Present address: Laboratory for Medical Microbiology, Ghent University Hospital, Ghent, Belgium. ${ }^{47}$ Present address: Advanced Light and Electron Microscopy (ZBS-4), Robert Koch Institute, Berlin, Germany. ${ }^{48}$ Present address: Quality Milk Production Services, Animal Health Diagnostic Center, Cornell University, Ithaca, NY, USA. ${ }^{49}$ Present address: Servicio de Microbiología, Complejo Asistencial Universitario de Salamanca, Salamanca, Spain. ${ }^{50}$ Present address: Department of Microbiology, Public Health Agency of Sweden, Solna, Sweden. ${ }^{51}$ Present address: Intervacc AB, Stockholm, Stockholm, Sweden. ${ }^{52}$ Present address: Department of Biomedical Science and Veterinary Public Health, Swedish University of Agricultural Sciences, Uppsala, Sweden. ${ }^{53}$ Present address: Antimicrobial Resistance and Healthcare Associated Infections Reference Unit, UK Health Security Agency, London, UK. ${ }^{54}$ Present address: Department of Physiology, Development \& Neuroscience, University of Cambridge, Cambridge, UK. ${ }^{55}$ These authors contributed equally: Jesper Larsen, Claire L. Raisen. ${ }^{56}$ These authors jointly supervised this work: Mark A. Holmes, Ewan M. Harrison, Anders R. Larsen. ${ }^{凶}$ e-mail: jrl@ssi.dk 


\section{Methods}

\section{Hedgehog survey}

The aim was to collect hedgehog samples covering the geographical range of hedgehogs in Europe and New Zealand. Personnel at 16 wildlife rescue centres in ten European countries and two wildlife rescue centres in New Zealand were instructed to obtain samples from the nasal area, skin and feet of hedgehogs kept in separate enclosures using FLOQSwabs (Copan). Swabs were stored in liquid Amies medium at ambient temperature and sent to the National Reference Laboratory for Antimicrobial Resistance at Statens Serum Institut in Denmark or the Department of Veterinary Medicine at University of Cambridge in the UK immediately after sample collection. For each swab, a loopful $(10 \mu \mathrm{l})$ of liquid Amies was inoculated into $5 \mathrm{ml}$ Mueller-Hinton broth (Oxoid) supplemented with $6.5 \% \mathrm{NaCl}$ and incubated overnight at $35-37^{\circ} \mathrm{C}$. A loopful $(10 \mu \mathrm{l})$ of enrichment broth was then streaked on a Brilliance MRSA 2 (Oxoid) agar plate followed by incubation at $35-37^{\circ} \mathrm{C}$ for $24 \mathrm{~h}$. One presumptive MRSA colony from each plate was subcultured on a blood agar plate at $35-37^{\circ} \mathrm{C}$ for $24 \mathrm{~h}$ and archived at $-80^{\circ} \mathrm{C}$. We also screened all MRSA-negative samples for the presence of MSSA isolates by streaking a loopful $(10 \mu \mathrm{l})$ of enrichment broth on a SaSelect (Bio-Rad) and Brilliance Staph 24 (Oxoid) agar plate followed by incubation at $35-37^{\circ} \mathrm{C}$ for $24 \mathrm{~h}$. One presumptive $S$. aureus colony from each plate was subcultured on a blood agar plate at $35-37^{\circ} \mathrm{C}$ for $24 \mathrm{~h}$ and archived at $-80^{\circ} \mathrm{C}$.

The hedgehog survey was conducted by staff members of the wildlife rescue centres who collected swab samples in connection with routine checks. In accordance with the Animal Welfare Act 1999 administered by theNewZealandMinistryforPrimaryIndustriesandDirective2010/63/EU of the European Parliament and of the Council of 22 September 2010 on the protection of animals used for scientific purposes, no ethical approval was required as sample collection did not cause pain, suffering, distress or lasting harm equivalent to, or higher than, that caused by the introduction of a needle in accordance with good veterinary practice or deprived the animal of usual care. Ethical review was undertaken at the Department of Veterinary Medicine, University of Cambridge (CR76).

\section{Bacterial isolates and whole-genome sequencing}

A list of the 1,157 isolates used in this study is provided in Supplementary Table 1 , including all of the mecC-MRSA $(n=222)$ and MSSA $(n=22)$ isolates identified in the hedgehog survey described above and an European collection of mecC-MRSA $(n=786)$ and MSSA $(n=127)$ isolates belonging to CC130, CC425 and CC1943, which constitute the most successful mecC-MRSA clones in Europe ${ }^{10,11,25}$. Most of the Danish isolates originated from two nationwide collections of mecC-MRSA and MSSA isolates recovered from hedgehogs and humans. The Danish collection of hedgehog isolates included $114 \mathrm{mecC}$-MRSA isolates collected from 188 hedgehogs in a previous study ${ }^{8}$. We re-examined the 74 MRSA-negative hedgehog samples for the presence of MSSA isolates using the method described above, which led to the inclusion of two MSSA isolates belonging to $\mathrm{CC} 130(n=1)$ and CC425 $(n=1)$ as well as six mecC-MRSA isolates belonging to $\mathrm{CC} 130(n=3)$ and $\mathrm{CC} 1943(n=3)$ that were missed in the original screening. The Danish collection of human isolates comprised 334 mecC-MRSA and 2 MSSA isolates that were collected from colonized or infected individuals between 1975 and 2016 as part of the national MRSA and $S$. aureus bacteraemia surveillance programmes. The remaining CC130, CC425 and CC1943 isolates were selected to represent the known geographical distribution (mainly western and central Europe) and host repertoire (mainly humans, cattle, sheep, goats and wild animals) of each clone. Whole-genome sequencing of the 1,157 isolates was performed on different Illumina platforms at the Wellcome Sanger Institute or at Statens Serum Institut. Short-read sequence data are available in the European Nucleotide Archive/NCBISequence Read Archive under BioProject IDs PRJEB15105,
PRJEB21015, PRJEB2655, PRJEB2755, PRJEB2756, PRJEB28206, PRJEB3174, PRJEB32898, PRJNA596428 and PRJEB43456 and the genome accession numbers are provided in Supplementary Table 1.

\section{Sequence analyses}

Draft genomes were de novo assembled using SPAdes (v.3.15) ${ }^{46}$. Multilocus sequence typing (MLST) was performed by comparing the draft genomes with the $S$. aureus MLST database ${ }^{47}$. We used the scn gene (which encodes staphylococcal complement inhibitor-A and an indicator of the IEC-1 element ${ }^{48}$ ) in S. aureus strain Newman (GenBank: NC_009641) and the $v w b_{\text {SaPI }}$ gene in SaPIbov4 (GenBank:HM211303) as queries in BLASTN searches against the draft genomes, setting length match to 0.9 and similarity match to 0.7 . These parameters were chosen to account for allelic diversity of the $v w b$ genes located on SaPIs (sharing $76-100 \%$ nucleotide identities with each other) ${ }^{49}$ while effectively excluding scn homologues located outside the IEC-1 element (sharing $48-61 \%$ nucleotide identities with $s c n)^{50}$. Contigs with hits for $v w b$ were analysed using PHASTER ${ }^{51}$ to ascertain that they were located on SaPIs. ARGs were detected by mapping sequence reads against the ResFinder database $\mathrm{e}^{52}$ using the $k$-mer alignment (KMA) tool (v.1.3 $)^{53}$, setting both length match and similarity match to 0.9 .

\section{Phylogenetic analyses of S. aureus CC130, CC425 and CC1943}

All 991 mecC-MRSA and 136 MSSA isolates belonging to CC130, CC425 and CC1943 were included. Mapping of sequence reads and SNP calling were performed using NASP (v.1.0 $)^{54}$ as follows: (1) sequence reads were mapped against the reference genome of $\operatorname{mec} C$-MRSA CC425 isolate LGA251 (GenBank: NC_017349) with the Burrows-Wheeler Alignment tool $^{55}$; (2) SNP calling was achieved using the GATK Unified Genotyper $^{56,57}$, setting depth of coverage and unambiguously base calls to $\geq 10 \times$ and $\geq 90 \%$, respectively, and ignoring insertions and deletions; and (3) SNPs contained in repeats were excluded using NUCmer ${ }^{58,59}$.

Unrooted maximum-likelihood phylogenetic trees were built from core-genome SNP alignments with PhyML (v.3.0) ${ }^{60,61}$ under the HKY85 substitution model after applying NNI moves to improve the BIONJ starting tree. Putative recombinogenic regions were detected and a recombination-corrected phylogeny was built with ClonalFrameML (v.1.12) ${ }^{62}$. Time-resolved phylogenies, in which the date of each node is estimated, were constructed with BactDating (v.1.0 ${ }^{63}$ using an additive uncorrelated relaxed clock model ${ }^{64}$. Convergence and mixing of the Markov Chain Monte Carlo chains were determined using the R package coda ${ }^{65}$. The time-resolved phylogenies were rooted as inferred with BactDating (v.1.0 ${ }^{63}$ to maximize the posterior probability of the tree. Treedater (v.0.5) ${ }^{66}$ was also applied for the same purpose and found to give similar estimates for the dating of nodes (Supplementary Figs. 6-11 and Source Data of Fig. 3).

The phylogenetic relationships between the type XI SCCmec elements were investigated in a separate analysis. A SNP alignment was generated from precalled SNPs from the $991 \mathrm{mecC}$-MRSA isolates. SNPS located outside the type XISCCmec element (corresponding to nucleotide positions 34,403 through 63,839 in mecC-MRSA CC425 isolate LGA251) were manually removed, and the remaining SNPs were used to construct an unrooted maximum-likelihood phylogenetic tree using PhyML (v.3.0) ${ }^{60,61}$ under the HKY85 substitution model after applying NNI moves to improve the BIONJ starting tree. Putative recombinogenic regions were detected and a recombination-corrected phylogeny was built using ClonalFrameML (v.1.12) ${ }^{62}$. The tips were manually mapped onto the CC130, CC425 and CC1943 phylogenies, and vice versa, to identify monophyletic mecC-MRSA lineages harbouring orthologous type XI SCCmec elements.

\section{Genome sequencing and analysis of the T.erinacei type strain IMI 101051}

DNA extracted from the T. erinacei type strain IMI 101051 was used to prepare a DNA library in accordance with the Nextera XT DNA Library 
Prep Guide (Illumina) and sequenced on a MiSeq platform (Illumina) with $2 \times 251$ bp using a MiSeq Reagent Kit v2 (Illumina). Short-read sequencing data were submitted to the European Nucleotide Archive under BioProject PRJEB43453. A draft genome was de novo assembled using SPAdes $(v .3 .15)^{46}$. We used the translated protein sequences of the $P$. chrysogenum $p c b A B, p c b C$ and $p e n D E$ genes and the $A$. chrysogenum cefD1, cefD2, cefEF and cefG genes (Extended Data Table1) as queries in TBLASTN searches against the draft genome. Hits were used as queries in BLASTP searches against the UniProtKB/Swiss-Prot protein database. Bidirectional best hits with a TBLASTN and BLASTP value of less than $1 \times 10^{-100}$ in both directions were considered as orthologous gene pairs.

\section{In vitro antibiotic production by the $T$. erinacei type strain IMI 101051}

In vitro antibiotic production by the T. erinacei type strain IMI 101051 was determined using a method modified from Smith and Marples ${ }^{15}$. In brief, the strain was grown on a Sabouraud dextrose agar plate (Oxoid) for 7 days at $30^{\circ} \mathrm{C}$. The culture was removed using distilled water and a $100-\mu$ l suspension was placed in $5 \mathrm{ml}$ Sabouraud dextrose broth (SDB) (Sigma-Aldrich) followed by incubation at $30^{\circ} \mathrm{C}$ with shaking at $200 \mathrm{rpm}$ for 3 days. The culture was placed on a Miracloth mesh (Calbiochem) in a 13- $\mathrm{cm}$ petri dish containing $35 \mathrm{ml} \mathrm{SDB}$. The plate was incubated at $30^{\circ} \mathrm{C}$ for 7 days, after which the mesh containing the fungal mass was placed in a $2-1$ conical flask containing $75 \mathrm{ml} \mathrm{SDB}$ supplemented with $2 \%$ glucose. The flask was incubated at $30^{\circ} \mathrm{C}$ with shaking at $200 \mathrm{rpm}$ for 7 days. The broth was replaced with fresh 5-strength $\mathrm{SDB}$, and the flask was incubated at $30^{\circ} \mathrm{C}$ for another 6 days. The culture medium was transferred into two 50-ml Falcon tubes containing $3 \mathrm{ml}$ Diaion HP-20 resin (Supelco) slurried in $3 \mathrm{ml}$ distilled water. The tubes were placed on a rotator for $1 \mathrm{~h}$, after which the beads were allowed to settle. The supernatant was discarded and antibiotics were eluted from the resin with $9 \mathrm{ml}$ acetone. The eluate was placed on a rotator for $30 \mathrm{~min}$ and centrifuged briefly to pellet the resin. The supernatant was transferred to 2-ml Eppendorf tubes, which were placed in a rotary evaporator at $30^{\circ} \mathrm{C}$. This process was repeated until all of the eluate had evaporated to dryness. To prepare bacterial inocula, S. aureus strains were individually grown overnight on blood agar plates (Oxoid) at $37^{\circ} \mathrm{C}$. Colonies were suspended in phosphate-buffered saline (PBS) to a 0.5 McFarland standard, diluted 1:10 in PBS, and streaked evenly on the surface of a Iso-Sensitest agar plate (Oxoid) using a sterile cotton-wool swab. Dried fungal culture extracts were suspended in $200 \mu$ l distilled water. Sterile paper discs were impregnated with $10 \mu \mathrm{l}$ solution and placed on the agar plates. In vitro antibiotic production was assessed by measuring the inhibition zones after overnight incubation at $35^{\circ} \mathrm{C}$.

\section{Metabolic profiling by LC-MS}

Dried fungal culture extracts were suspended in $50 \%$ methanol to a concentration of $100 \mathrm{mg} \mathrm{ml}^{-1}$, diluted 1:10 in pure methanol and analysed using LC-MS. Metabolic profiles were obtained on a Vanquish UHPLC system (Thermo Fisher Scientific) coupled to a Vanquish diode array detector (Thermo Fisher Scientific) and an Orbitrap Fusion Tribrid high-resolution tandem mass spectrometer (Thermo Fisher Scientific). Chromatographic separation of $5 \mu$ l fungal culture extracts was performed on a Luna C18 column $(3 \mu \mathrm{m}, 3 \times 150 \mathrm{~mm})$ (Phenomenex) using a linear mobile phase gradient from $0 \%$ methanol, $90 \%$ water and $10 \%$ acetonitrile containing $1 \%(\mathrm{v} / \mathrm{v})$ formic acid to $90 \%$ methanol, $0 \%$ water and $10 \%$ acetonitrile containing $1 \%(\mathrm{v} / \mathrm{v})$ formic acid over

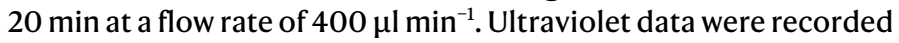
between $210 \mathrm{~nm}$ and $550 \mathrm{~nm}$. For comparative purposes, a pure standard of penicillin $\mathrm{G}$ (Sigma-Aldrich) was prepared at a final concentration of $9.4 \mu \mathrm{g} \mathrm{ml}^{-1}$ and analysed using LC-MS.

Mass spectra were obtained in the positive and negative ionization modes using the full scan and data-dependent $\mathrm{MS}^{2}$ and $\mathrm{MS}^{3}$ acquisition modes. Full scan total ion current chromatograms were obtained over the range of $125-1,800 \mathrm{~m} / z$ using a spray voltage of $+3.5 \mathrm{kV}$ and
$-2.5 \mathrm{kV}$ for the positive and negative ionization modes, respectively. Three different scan events were recorded for the data-dependent acquisition modes as follows: (1) $\mathrm{MS}^{2}$ of the most intense ion in the full scan acquisition mode; (2) $\mathrm{MS}^{3}$ of the most intense ion in the $\mathrm{MS}^{2}$ spectra; and (3) $\mathrm{MS}^{3}$ of the second-most intense ion in the $\mathrm{MS}^{2}$ spectra. Additional parameters for the MS included the following: full scan resolution set to 60,000 (full-width at half-maximum, FWHM), capillary temperature set to $350{ }^{\circ} \mathrm{C}$, ion transfer tube temperature set to $325^{\circ} \mathrm{C}$, RF lens set to $50 \%$, automatic gain control target set to $4.0 \times 10^{5}$ (full scan) or $1.0 \times 10^{4}\left(\mathrm{MS}^{2}\right.$ and $\left.\mathrm{MS}^{3}\right)$, intensity threshold set to $1.0 \times 10^{4}$, collision-induced dissociation energy set to $35 \mathrm{eV}$, activation $Q$ set to 0.25 and isolation window set to $4 \mathrm{~m} / z$. Nitrogen was used as the drying, nebulizer and fragmentation gas.

\section{Molecular networking analysis of LC-MS data}

A molecular network was created using the Global Natural Products Social Molecular Networking (GNPS) workflow ${ }^{67}$. Chromatographic raw data in the positive ionization mode were transformed to the mzXML format using the ProteoWizard command-line utility msConvert ${ }^{68}$. The data were then filtered by removing all $\mathrm{MS}^{2}$ fragment ions within \pm 17 Da of the precursor $m / z$ value. The precursor ion mass and $\mathrm{MS}^{2}$ fragment ion tolerances were set to 2.0 Da and 0.5 Da, respectively, to enable more comprehensive comparisons with the GNPS database. $\mathrm{MS}^{2}$ spectra were filtered by choosing the top six fragment ions in the \pm 50 -Da window throughout the spectrum. A network was then created considering a cosine score above 0.7 and more than six matched peaks to link different nodes through edges. Edges between two nodes were kept in the network if each of the nodes appeared in each other's respective top ten most similar nodes. Finally, the maximum size of a molecular family was set to 100 . The spectra in the network were then searched against spectral libraries in GNPS, filtering the library spectra in the same manner as the input data. All matches between network spectra and library spectra were required to have a score above 0.7 and at least six matched peaks.

\section{Statistics}

Statistical analyses were performed using GraphPad Prism (v.8.3) (GraphPad Software). We used two-tailed paired Student's $t$-tests to compare inhibition zones of each mutant to the corresponding mecC-MRSA wild-type strain and a two-tailed Wilcoxon matched-pairs signed rank test to compare population structures of mecC-MRSA at the lineage level.

\section{Reporting summary}

Further information on research design is available in the Nature Research Reporting Summary linked to this paper.

\section{Data availability}

LC-MS data in mzXML format and molecular networking results are available at the MassIVE repository under identifier MSV000087335. S. aureus short-read sequence data have been deposited in the European Nucleotide Archive/NCBI Sequence Read Archive under BioProject IDs PRJEB15105, PRJEB21015, PRJEB2655, PRJEB2755, PRJEB2756, PRJEB28206, PRJEB3174, PRJEB32898, PRJNA596428 and PRJEB43456 and the genome accession numbers are provided in Supplementary Table 1. T. erinacei type strain IMI 101051 short-read sequence data have been deposited in the European Nucleotide Archive under BioProject PRJEB43453. Tree and genealogy files in Newick format are provided in Supplementary Data 1-10. Source data are provided with this paper.

46. Bankevich, A. et al. SPAdes: a new genome assembly algorithm and its application to single-cell sequencing. J. Comput. Biol. 19, 455-477 (2012).

47. Enright, M. C., Day, N. P., Davies, C. E., Peacock, S. J., Spratt, B. G. Multilocus sequence typing for characterization of methicillin-resistant and methicillin-susceptible clones of Staphylococcus aureus. J. Clin. Microbiol. 38, 1008-1015 (2000). 
48. Van Wamel, W. J., Rooijakkers, S. H., Ruyken, M. van Kessel, K. P. \& Strijp, J. A. The innate immune modulators staphylococcal complement inhibitor and chemotaxis inhibitory protein of Staphylococcus aureus are located on beta-hemolysin-converting bacteriophages. J. Bacteriol. 188, 1310-1315 (2006).

49. Viana, D. et al. Adaptation of Staphylococcus aureus to ruminant and equine hosts involved SaPI-carried variants of von Willebrand factor-binding protein. Mol. Microbiol. 77, 1583-1594 (2010).

50. Rooijakkers, S. H. M. et al. Staphylococcal complement inhibitor: structure and active sites. J. Immunol. 179, 2989-2998 (2007).

51. Arndt, D. et al. PHASTER: a better, faster version of the PHAST phage search tool. Nucleic Acids Res. 44, W16-W21 (2016)

52. Bortolaia, V. et al. ResFinder 4.0 for predictions of phenotypes from genotypes. J. Antimicrob. Chemother. 75, 3491-3500 (2020).

53. Clausen, P. T. L. C., Aarestrup, F. M. \& Lund, O. Rapid and precise alignment of raw reads against redundant database with KMA. BMC Bioinform. 19, 397 (2018).

54. Sahl, J. W. et al. NASP: an accurate, rapid method for the identification of SNPs in WGS datasets that supports flexible input and output formats. Microb. Genom. 2, e000074 (2016).

55. Li, H. \& Durbin, R. Fast and accurate short read alignment with Burrow-Wheeler transform Bioinformatics 25, 1754-1760 (2009)

56. McKenna, A. et al. The Genome Analysis Toolkit: a MapReduce framework for analyzing next-generation DNA sequencing data. Genome Res. 20, 1297-1303 (2010).

57. DePristo, M. A. et al. A framework for variation discovery and genotyping using next-generation sequencing data. Nat. Genet. 43, 491-498 (2011).

58. Delcher, A. L., Phillippy, A., Carlton, J. \& Salzberg, S. L. Fast algorithms for large-scale genome alignment and comparison. Nucleic Acids Res. 30, 2478-2483 (2002).

59. Kurz, S. et al. Versatile and open software for comparing large genomes. Genome Biol. 5 R12 (2004).

60. Guindon, S. \& Gasquel, O. A simple, fast, and accurate algorithm to estimate large phylogenies by maximum likelihood. Syst. Biol. 52, 696-704 (2003).

61. Guindon, S. et al. New algorithms and methods to estimate maximum-likelihood phylogenies: assessing the performance of PhyML 3.0. Syst. Biol. 59, 307-321 (2010).

62. Didelot, X. \& Wilson, D. J. ClonalFrameML: efficient inference of recombination in whole bacterial genome. PLoS Comput. Biol. 11, e1004041 (2015).

63. Didelot, X. et al. Bayesian inference of ancestral dates on bacterial phylogenetic trees. Nucleic Acids Res. 46, e134 (2018).

64. Didelot, X., Siveroni, I. \& Volz, E. M. Additive uncorrelated relaxed clock models for the dating of genomic epidemiology phylogenies. Mol. Biol. Evol. 38, 307-317 (2021).

65. Plummer, M., Best, N., Cowles, K. \& Vines, K. CODA: convergence diagnosis and output analysis for MCMC. R News 6, 7-11 (2006).

66. Volz, E. M. \& Frost, S. D. Scalable relaxed clock phylogenetic dating. Virus Evol. 3, vexO25 (2017).

67. Wang, M. et al. Sharing and community curation of mass spectrometry data with Global Natural Products Social Molecular Networking. Nat. Biotechnol. 34, 828-837 (2016).
68. Adusumilli, R. \& Mallick, P. Data conversion with ProteoWizard msConvert. Methods Mol. Biol. 1550, 339-368 (2017).

Acknowledgements We thank M. Ganoti, F. Attila-Zoltán, M. Dugar, Z. Pokorná, D. Madsen, S. M. Gamborg, R. Molina-López, C. Rodrigues, T. Vieira, J. Viricel, A. Fingar, K. South, G. Prince, H. Gasser, S. Sebright, N. Ennew, C. Catchpole, E. Acton, N. Acton, K. Horrey and P. Loague for providing hedgehog samples; A. Medina, L. R. H. Kildevang, P. T. Hansen, and S. M. Johansson for technical assistance during the analysis of hedgehog samples; A. E. Henius for help with Figs. 1, 4 and Extended Data Fig. 8; and V. Bortolaia for reading the manuscript. This work is dedicated to V. R. Simpson, who passed away during the study. B.Č.B. was supported by a grant from the Czech University of Life Sciences Prague (no. IGA 20213106). X.D. was funded by a grant from the National Institute for Health Research (NIHR) Health Protection Research Unit in Genomics and Enabling Data (no. NIHR200892). M.A.H. was supported by grants from the Medical Research Council (nos. G1001787/1, MR/NO02660/1 and MR/P007201/1) and the Economic and Social Research Council (no. ES/S000186/1). E.M.H. was supported by a UK Research and Innovation (UKRI) Fellowship (no. MR/S00291X/1). We acknowledge Eurostat for the provision of maps, which are licensed under the Creative Commons Attribution 4.0 International (CC BY 4.0) licence, and administrative boundaries, which are copyright of EuroGeographics.

Author contributions J.L., M.A.H., E.M.H. and A.R.L. initiated and designed the study. J.L., C.L.R., B.Č.B., P.H. and S.L.R. directed the hedgehog survey. J.L., C.L.R. X.B. and Ø.A. analysed hedgehog samples. C.L.R., I.L., H.K., P.A., R.H., A.D., S.V., R.L.S., A.P., C.E.-G., F.M.A., L.J.L., S.M.N., F. Laurent, K.B., B.W., C.K., C.C., F. Layer, G.W., W.W., I.S., P.M., H.J.J., H.d.L., E.C., F.G.-G., S.B., S.H., V.P., C.J.T., A.S.W., B.P., M.D.C., M.J.E., S.J.P., D.J.S., N.F.H., J.A.L., G.F.E., G.F., G.K.P., M.A.H. and A.R.L. provided bacterial isolates from other sources. X.B., C.B., M.S., F.J.E.M., G.K.P. and M.A.H. conducted whole-genome sequencing of bacterial isolates. J.L., C.L.R., R.N.S., J.J.W., J.P., M.T.G.H., X.D., M.A.H. and E.M.H. performed sequence and phylogenetic analyses of bacterial isolates. J.L., M.C.A. and R.K.H. performed genome sequencing and analysis of T. erinacei IMI 101051. C.L.R., X.B. and D.J.S. investigated in vitro antibiotic production by T. erinacei IMI 101051. N.J.S. prepared samples, performed LC-MS analysis and guided the identification of the penicillin-like substances. G.F.P.-G. analysed LC-MS data, performed molecular networking analysis and identified the two penicillin-like substances. M.S.J.S. coordinated and supervised the LC-MS analysis. J.L. wrote the manuscript with considerable input from C.L.R., X.B., N.J.S., G.F.P.-G., X.D., M.A.H., E.M.H. and A.R.L. All of the authors reviewed the manuscript.

Competing interests The authors declare no competing interests.

Additional information

Supplementary information The online version contains supplementary material available at https://doi.org/10.1038/s41586-021-04265-w.

Correspondence and requests for materials should be addressed to Jesper Larsen.

Peer review information Nature thanks Ross Fitzgerald and the other, anonymous, reviewer(s) for their contribution to the peer review of this work.

Reprints and permissions information is available at http://www.nature.com/reprints. 


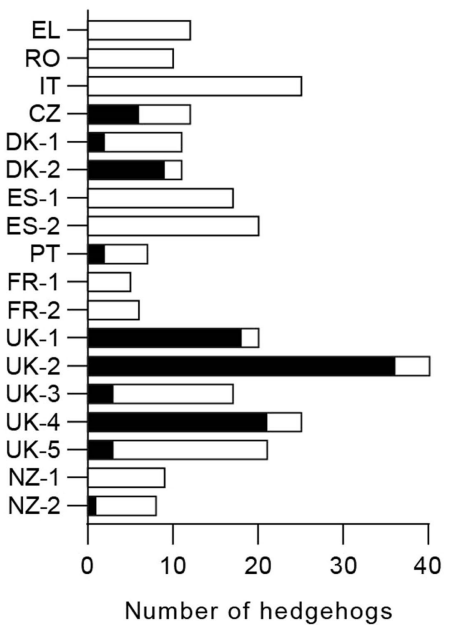

Attica, Greece (EL)

Județul Cluj, Romania (RO)

Treviso, Italy (IT)

Prague, Czech Republic (CZ)

Jutland, Denmark (DK-1)

Zealand, Denmark (DK-2)

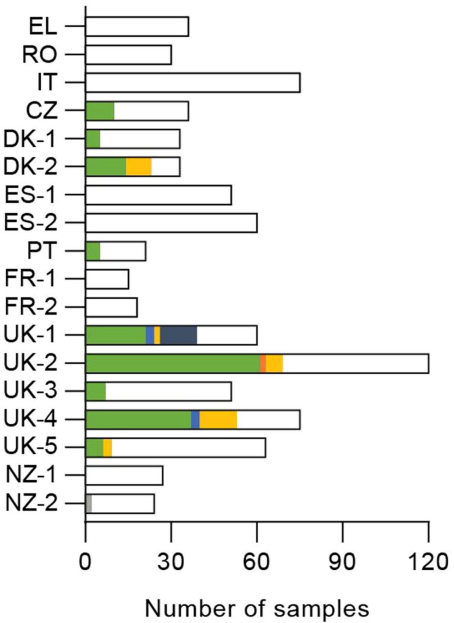

Valencia, Spain (ES-1)

Catalunya, Spain (ES-2)

Norte Region, Portugal (PT)

Loire-sur-Rhône, France (FR-1)

Fouencamps, France (FR-2)

Cornwall, England (UK-1)

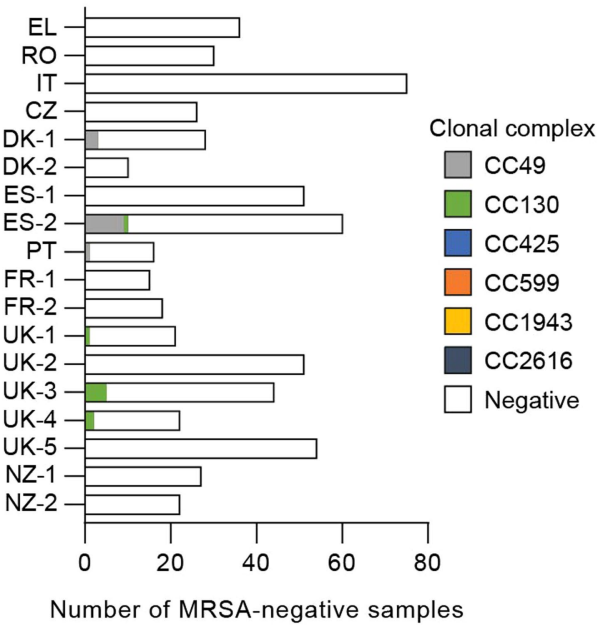

Berkshire, England (UK-2)

Cambridgeshire, England (UK-3)

Northumberland, England (UK-4)

Powys, Wales (UK-5)

South Island, New Zealand (NZ-1)

North Island, New Zealand (NZ-2)
Extended Data Fig. 1 | Distribution of mecC-MRSA clones in European and New Zealand hedgehogs. The analysis included 828 samples from the nasal area, skin and feet of 276 hedgehogs originating from 16 wildlife rescue centres in ten European countries and two wildlife rescue centres in New Zealand. a, Presence of mecC-MRSA in hedgehogs $(n=276)$. Presence and absence are shown as black and white boxes, respectively. $\mathbf{b}$, Distribution of mecC-MRSA clones in hedgehog samples $(n=828)$. c, Distribution of MSSA clones in MRSA-negative hedgehog samples $(n=606)$. 
a
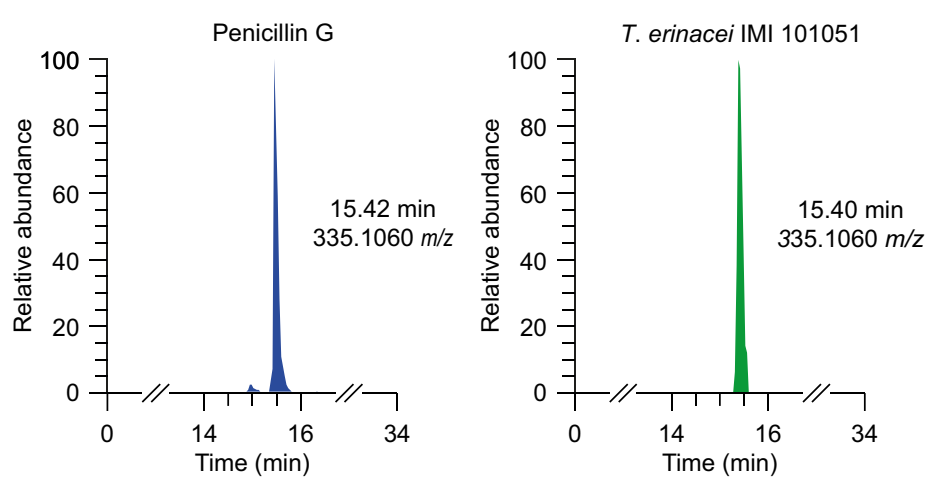

b

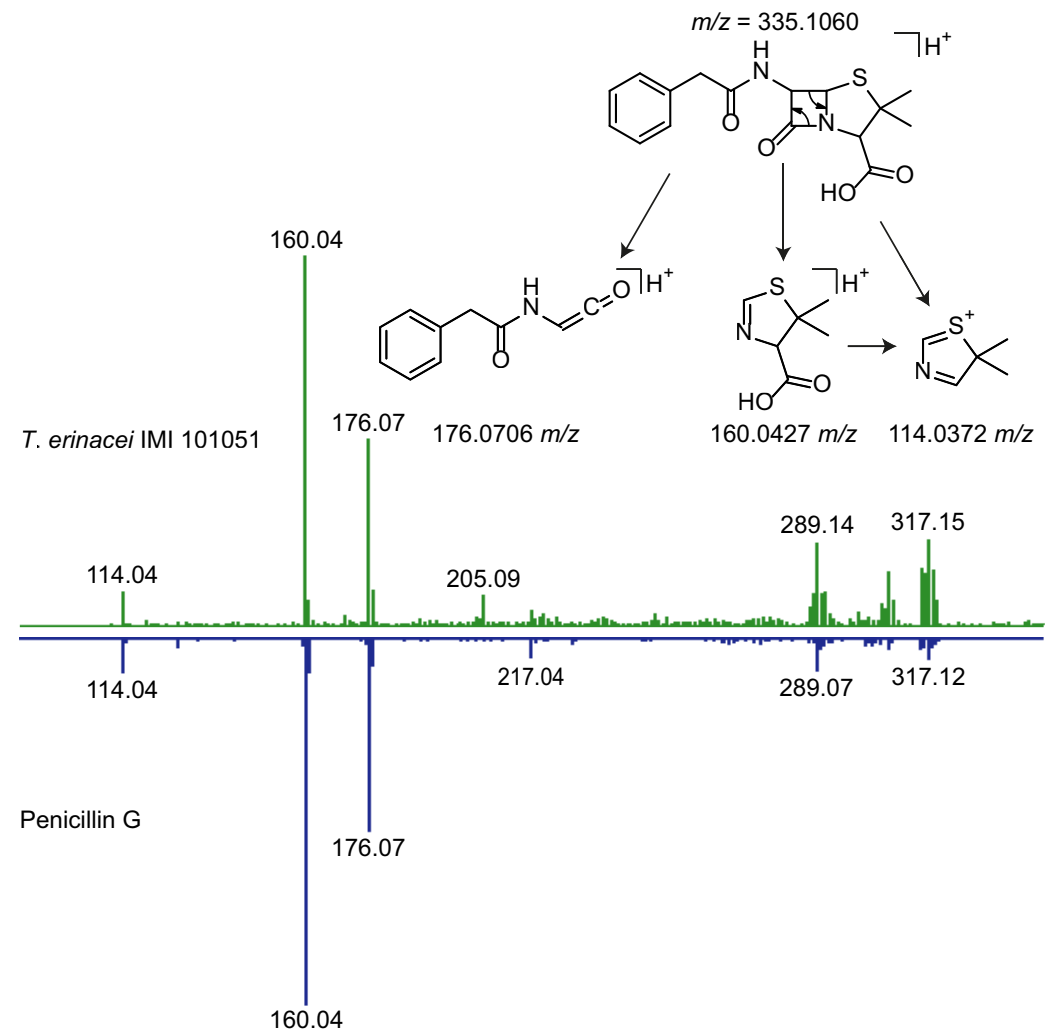

Extended Data Fig. 2 | Detection of penicillin G in T. erinacei IMI 101051 culture broth. a, Left and right panels show extracted ion chromatogram of a pure standard of penicillin $\mathrm{G}$ and in T. erinacei culture broth, respectively. b, Upper and lower panels show $\mathrm{MS}^{2}$ spectra of penicillin $\mathrm{G}$ in a T.erinacei culture broth and a pure standard of penicillin $\mathrm{G}$, respectively. 
a
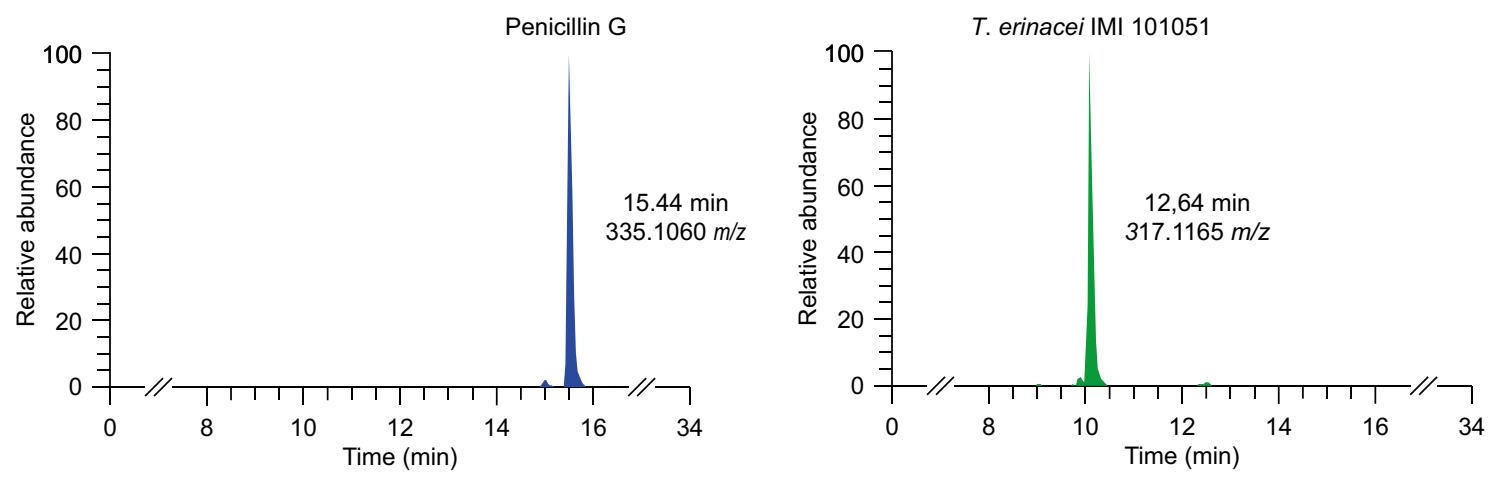

b

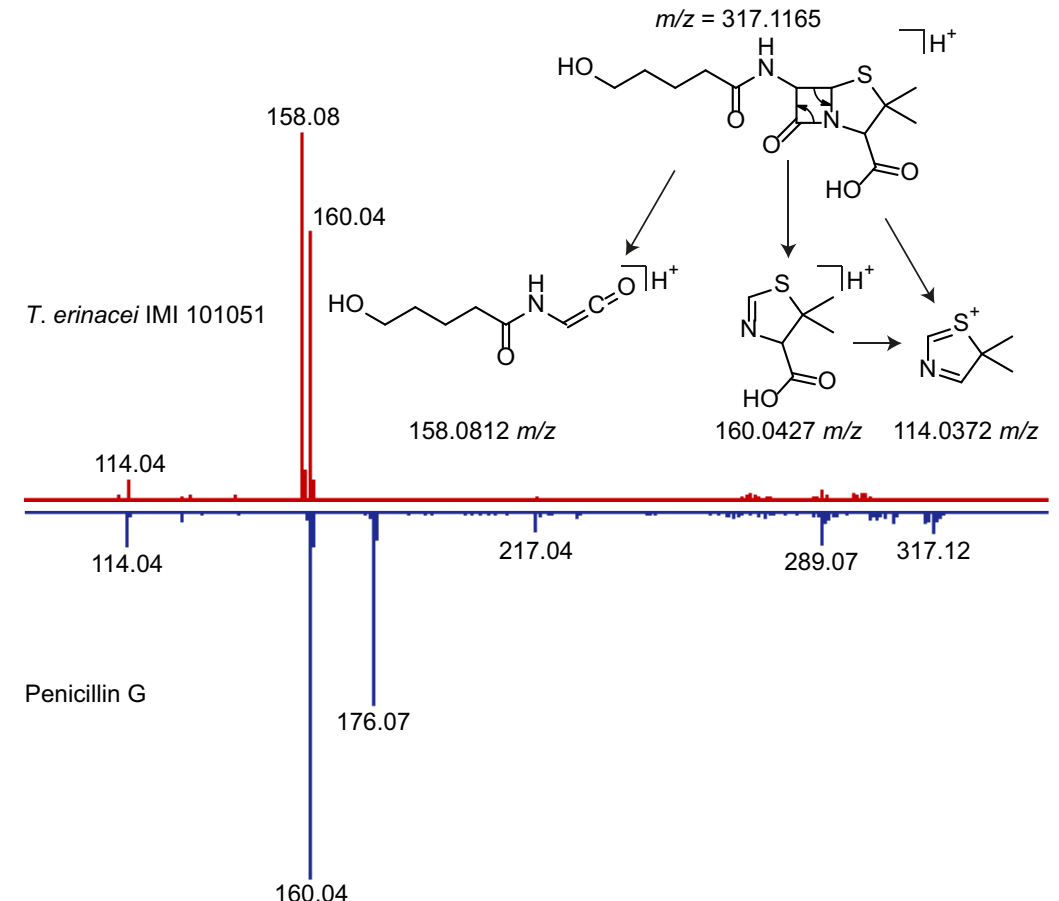

Extended Data Fig. 3 | Detection of KPN in T. erinacei IMI 101051 culture broth. a, Left and right panels show extracted ion chromatogram of a pure standard of penicillin G and KPN in T. erinacei culture broth, respectively. b, Upper and lower panels show $\mathrm{MS}^{2}$ spectra of KPN in a T. erinacei culture broth and a pure standard of penicillin $\mathrm{G}$, respectively. 


\section{Article}
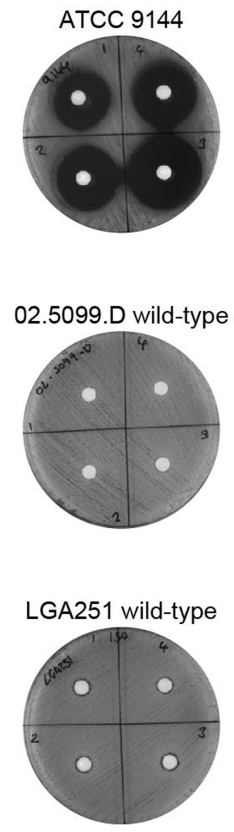

Extended Data Fig. 4 | Antibiotic activity of T. erinacei IMI 101051. T. erinacei inhibition zones against a collection of $S$. aureus control strains and two mecC-MRSA wild-type strains belonging to CC130 (02.5099.D) and CC425
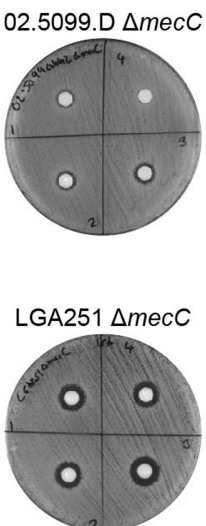
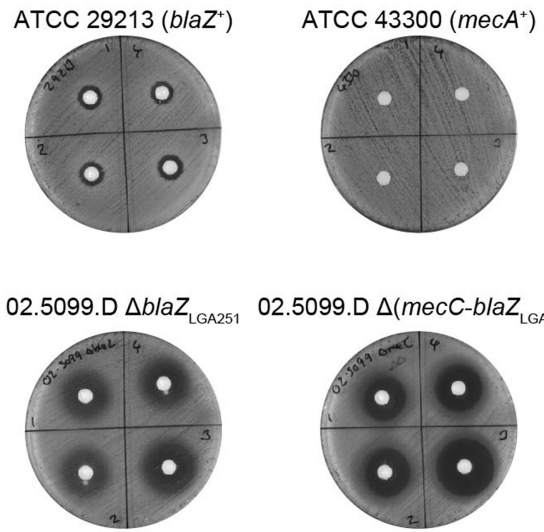

02.5099.D $\Delta\left(\right.$ mecC-blaZ $\left.{ }_{\mathrm{LGA251}}\right)$
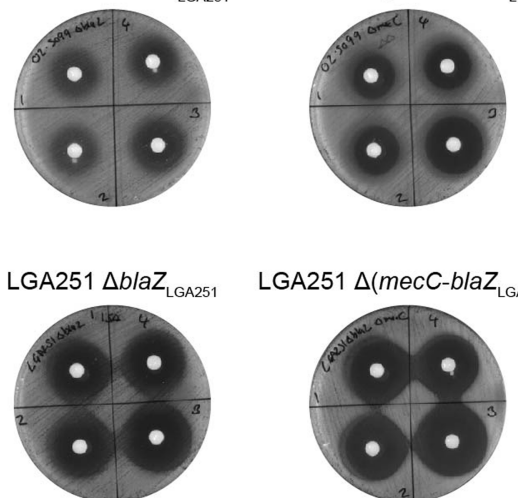

LGA251 $\Delta\left(\right.$ mecC-blaZ $\left.Z_{\text {LGA251 }}\right)$

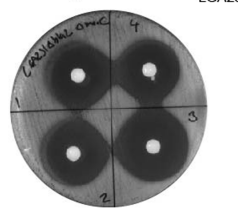

(LGA251) and their isogenic mutants. The numbers on the plates refer to each of four biologically independent fungal culture extracts. 


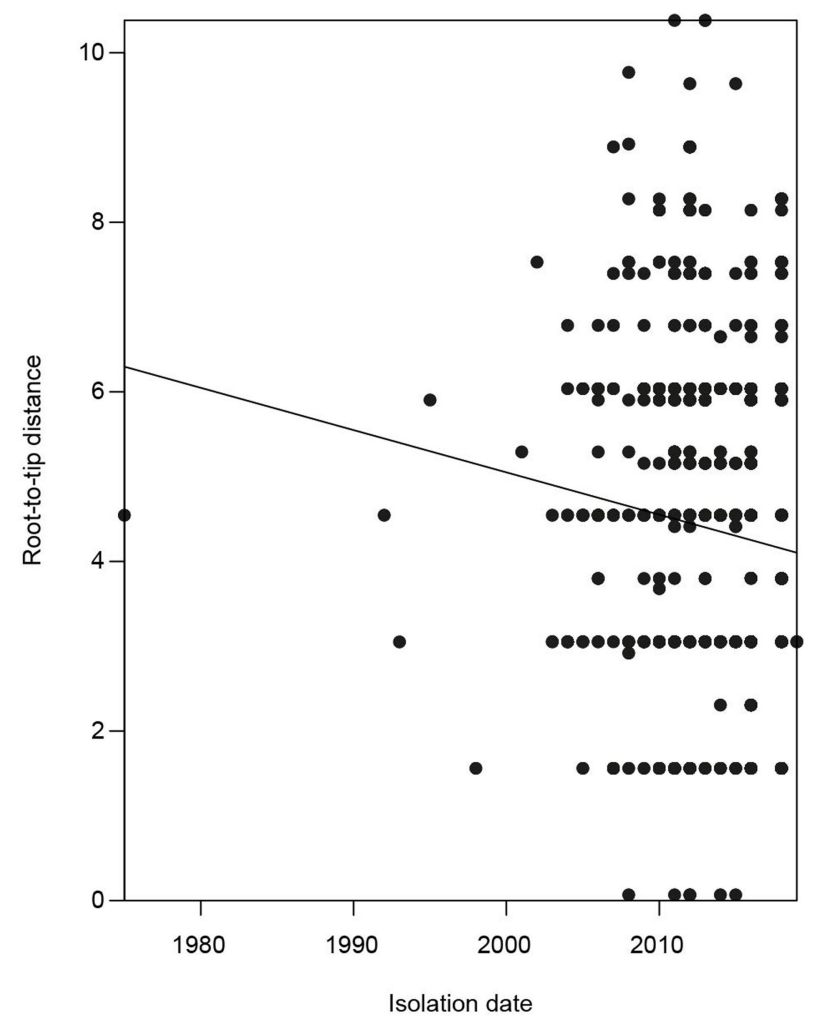

Extended Data Fig. 5 | Root-to-tip linear regression analysis of the type XI SCCmec dataset. The correlation between root-to-tip distances and isolation dates is very weak with a coefficient of determination $R^{2}=-0.05$. 


\section{Article}

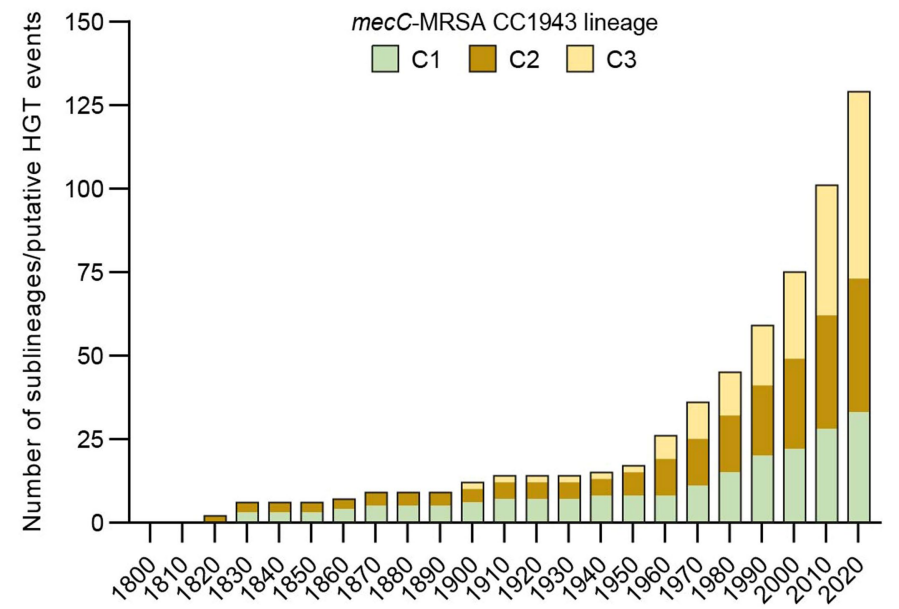

Extended Data Fig. 6 | Number of mecC-MRSA CC1943 sublineages. The bars show the number of sublineages of mecC-MRSA CC1943:C1, mecC-MRSA CC1943:C2 and mecC-MRSA CC1943:C3 at different time points. 


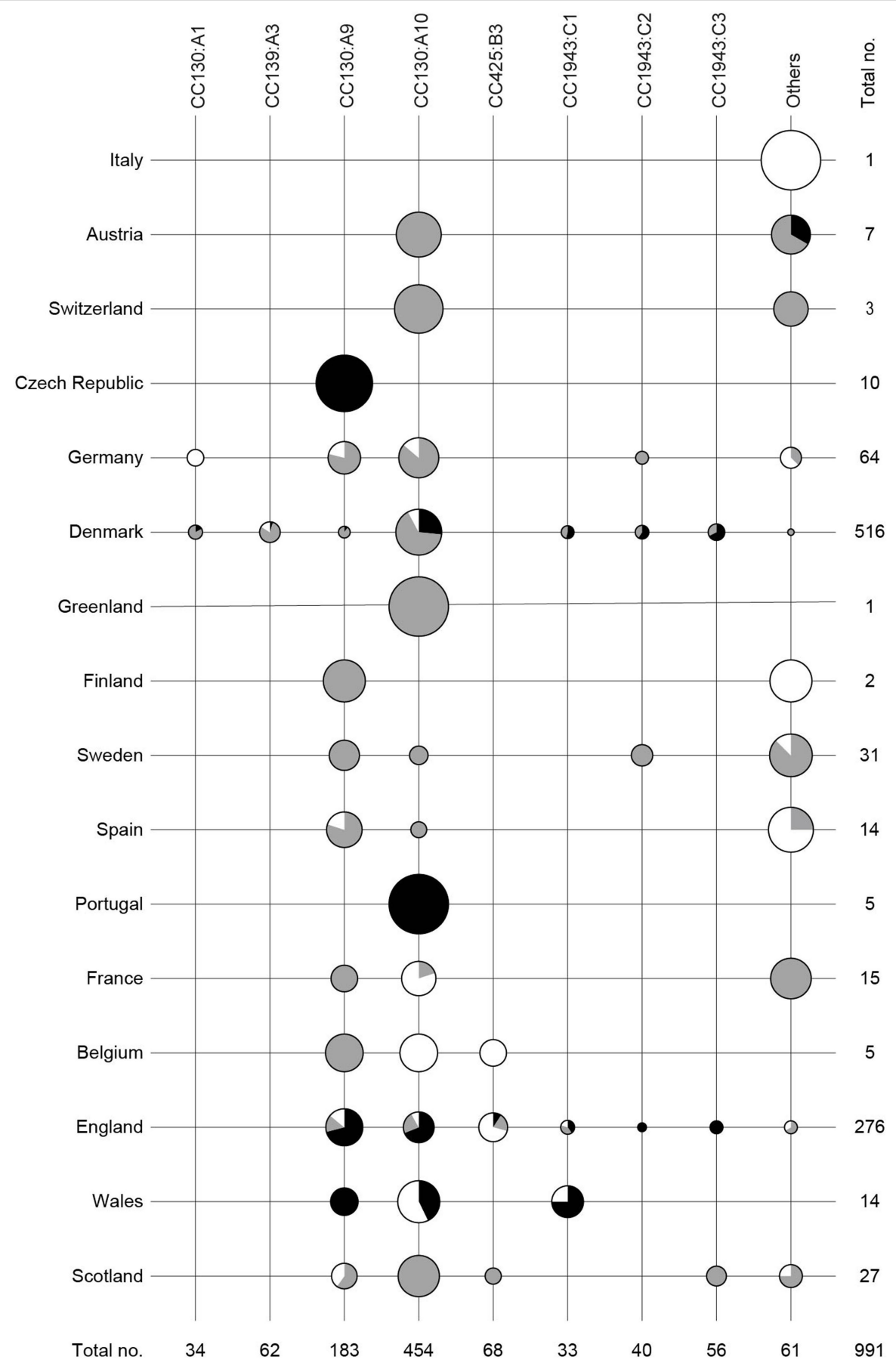

Extended Data Fig. 7 | Geographical distribution and host range of major mecC-MRSA CC130, CC425 and CC1943 lineages. The study collection included $991 \mathrm{mecC}$-MRSA CC130, CC425 and CC1943 isolates originating from 16 European countries. The countries of sampling and the eight largest $(\geq 25$ isolates) mecC-MRSA lineages are shown. Isolates belonging to the eight minor

mecC-MRSA lineages are grouped together (others). The pie charts depict the proportion of mecC-MRSA isolates from hedgehogs (black), humans (grey) and other sources (white). The area of the pie chart is proportional to the number of mecC-MRSA isolates from that country. 


\section{Article}

a

a

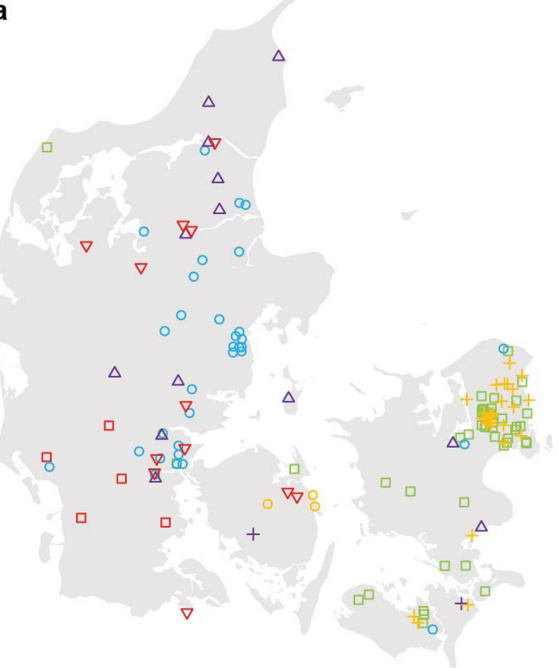

Extended Data Fig. 8 |Sampling locations of Danish mecc-MRSA isolates

from hedgehogs and humans. The maps of Denmark relate to the map shown in Fig. 4. a, mecC-MRSA recovered from hedgehogs $(n=141)$. b, mecC-MRSA

recovered from humans $(n=327)$. The location of each sample is given at the zip b

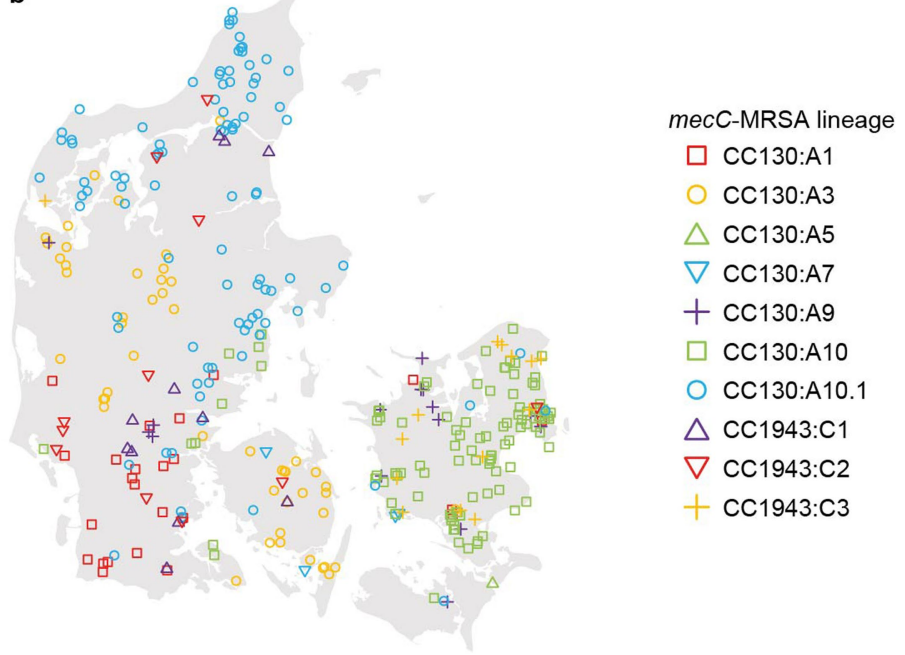

code area level. Maps were provided by Eurostat under a Creative Commons Attribution 4.0 International (CC BY 4.0) licence; the administrative boundaries are copyright of EuroGeographics. 


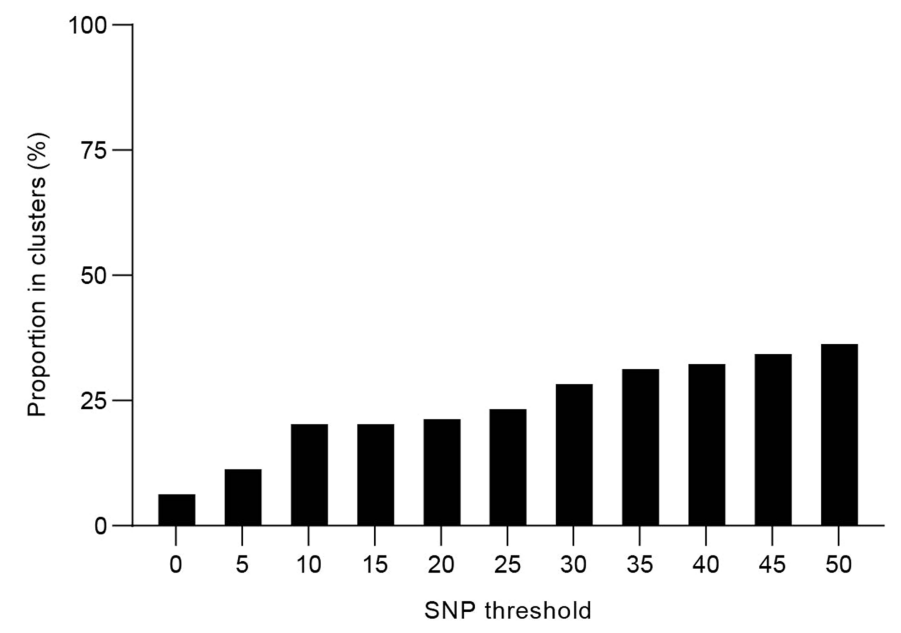

Extended Data Fig. 9 | Frequency of potential transmission events of mecC-MRSA CC130 isolates within wildlife rescue centres. The bars show the proportion of isolates that belong to clusters defined at different pairwise SNP distance thresholds. 


\section{Article}

Extended Data Table 1 | Penicillin biosynthetic genes in T. erinacei IMI 101051

\begin{tabular}{|c|c|c|c|c|}
\hline \multicolumn{2}{|c|}{ Best TBLASTN hit in $T$. erinacei IMI 101051 draft genome } & \multicolumn{3}{|c|}{ Best BLASTP hit in UniProtKB/Swiss-Prot } \\
\hline Query (accession no.) & E-value & E-value & Hit (accession no.)* & Pathway \\
\hline pcbAB (P19787) & 0 & 0 & $p c b A B(\mathrm{P} 19787)$ & Penicillin $\mathrm{G}$ biosynthesis \\
\hline$p c b C(\mathrm{P} 08703)$ & $6.38-178$ & 0 & $p c b C$ (P08703) & Penicillin G biosynthesis \\
\hline penDE (P15802) & $1.96 \mathrm{e}-124$ & $2.95 e-133$ & penDE (P21133) & Penicillin G biosynthesis \\
\hline cefD1 (Q8J0E9) & $2.15 e-157$ & $4.44 \mathrm{e}-174$ & cefD1 (Q8J0E9) & Cephalosporin $\mathrm{C}$ biosynthesis \\
\hline cefD2 (Q8J0F0) & $3.40 \mathrm{e}-143$ & $5.21 \mathrm{e}-160$ & cefD2 (Q8J0F0) & Cephalosporin $\mathrm{C}$ biosynthesis \\
\hline cefEF (P11935) & $9.62 \mathrm{e}-07$ & $1.21 \mathrm{e}-32$ & ppzD (A0A166YZY4) & Secondary metabolite biosynthesis \\
\hline cefG (P39058) & $1.56 e-81$ & 0 & metE (Q9Y875) & L-methionine biosynthesis via de novo pathway \\
\hline
\end{tabular}

*The in silico identified putative $T$. erinacei penicillin $\mathrm{G}$ and cephalosporin $\mathrm{C}$ biosynthetic genes are shown in green 


\section{Reporting Summary}

Nature Research wishes to improve the reproducibility of the work that we publish. This form provides structure for consistency and transparency in reporting. For further information on Nature Research policies, see our Editorial Policies and the Editorial Policy Checklist.

\section{Statistics}

For all statistical analyses, confirm that the following items are present in the figure legend, table legend, main text, or Methods section.

$\mathrm{n} / \mathrm{a}$ Confirmed

$\bigotimes$ The exact sample size $(n)$ for each experimental group/condition, given as a discrete number and unit of measurement

$\bigotimes$ A statement on whether measurements were taken from distinct samples or whether the same sample was measured repeatedly

$\$ The statistical test(s) used AND whether they are one- or two-sided

Only common tests should be described solely by name; describe more complex techniques in the Methods section.

Х A description of all covariates tested

Х $\square$ A description of any assumptions or corrections, such as tests of normality and adjustment for multiple comparisons

$\triangle$ A full description of the statistical parameters including central tendency (e.g. means) or other basic estimates (e.g. regression coefficient) AND variation (e.g. standard deviation) or associated estimates of uncertainty (e.g. confidence intervals)

For null hypothesis testing, the test statistic (e.g. $F, t, r$ ) with confidence intervals, effect sizes, degrees of freedom and $P$ value noted Give $P$ values as exact values whenever suitable.

Х $\square$ For Bayesian analysis, information on the choice of priors and Markov chain Monte Carlo settings

$\bigotimes \square$ For hierarchical and complex designs, identification of the appropriate level for tests and full reporting of outcomes

$\bigotimes \square$ Estimates of effect sizes (e.g. Cohen's $d$, Pearson's $r$ ), indicating how they were calculated

Our web collection on statistics for biologists contains articles on many of the points above.

\section{Software and code}

Policy information about availability of computer code

Data collection

No software was used to collect data in our study.

Data analysis SPAdes V3.15

PHASTER

k-mer alignment (KMA) tool v1.3

NASP V1.0

PhyML V3.0

ClonalFrameML V1.12

BactDating v1.0

Treedater 0.5

coda

msConvert

GraphPad Prism v8.3

For manuscripts utilizing custom algorithms or software that are central to the research but not yet described in published literature, software must be made available to editors and

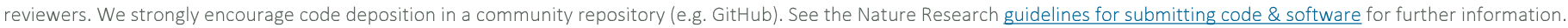


Policy information about availability of data

All manuscripts must include a data availability statement. This statement should provide the following information, where applicable:

- Accession codes, unique identifiers, or web links for publicly available datasets

A list of figures that have associated raw data

A description of any restrictions on data availability

LC-MS data in MZXML format and molecular networking results are available in the MassIVE repository with the identifier MSV000087335. S. aureus short-read sequence data have been deposited in the European Nucleotide Archive/NCBI Sequence Read Archive under BioProjects PRJEB15105, PRJEB21015, PRJEB2655,

PRJEB2755, PRJEB2756, PRJEB28206, PRJEB3174, PRJEB32898, PRJNA596428, and PRJEB43456 and the genome accession numbers are provided in Supplementary

Table 1. T. erinacei type strain IMI 101051 short-read sequence data have been deposited in the European Nucleotide Archive under BioProject PRJEB43453. Tree and

genealogy files in Newick format are provided in Supplementary Data 1-10. Source data for Figs. 1-4 and Extended Data Figs. 1, 4, 6, 7, and 9 are provided with the paper.

\section{Field-specific reporting}

Please select the one below that is the best fit for your research. If you are not sure, read the appropriate sections before making your selection.

Life sciences

Behavioural \& social sciences

Ecological, evolutionary \& environmental sciences

For a reference copy of the document with all sections, see nature.com/documents/nr-reporting-summary-flat.pdf

\section{Life sciences study design}

All studies must disclose on these points even when the disclosure is negative.
Sample size
Describe how sample size was determined, detailing any statistical methods used to predetermine sample size OR if no sample-size calculation was performed, describe how sample sizes were chosen and provide a rationale for why these sample sizes are sufficient.
Data exclusions Describe any data exclusions. If no data were excluded from the analyses, state so OR if data were excluded, describe the exclusions and the rationale behind them, indicating whether exclusion criteria were pre-established. $O R$ if there are any findings that were not replicated or cannot be reproduced, note this and describe why.

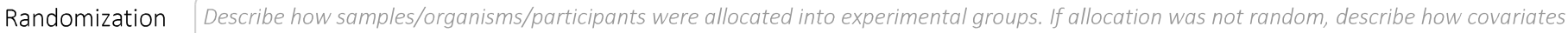 were controlled $O R$ if this is not relevant to your study, explain why.

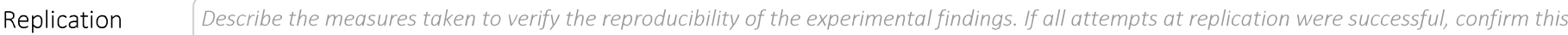

Blinding Describe whether the investigators were blinded to group allocation during data collection and/or analysis. If blinding was not possible, describe why OR explain why blinding was not relevant to your study.

\section{Behavioural \& social sciences study design}

All studies must disclose on these points even when the disclosure is negative.

Study description

Research sample

Sampling strategy

Data collection

Timing

Data exclusions
Briefly describe the study type including whether data are quantitative, qualitative, or mixed-methods (e.g. qualitative cross-sectional, quantitative experimental, mixed-methods case study).

State the research sample (e.g. Harvard university undergraduates, villagers in rural India) and provide relevant demographic information (e.g. age, sex) and indicate whether the sample is representative. Provide a rationale for the study sample chosen. For studies involving existing datasets, please describe the dataset and source.

Describe the sampling procedure (e.g. random, snowball, stratified, convenience). Describe the statistical methods that were used to predetermine sample size OR if no sample-size calculation was performed, describe how sample sizes were chosen and provide a rationale for why these sample sizes are sufficient. For qualitative data, please indicate whether data saturation was considered, and what criteria were used to decide that no further sampling was needed.

Provide details about the data collection procedure, including the instruments or devices used to record the data (e.g. pen and paper, computer, eye tracker, video or audio equipment) whether anyone was present besides the participant(s) and the researcher, and whether the researcher was blind to experimental condition and/or the study hypothesis during data collection.

Indicate the start and stop dates of data collection. If there is a gap between collection periods, state the dates for each sample cohort.

If no data were excluded from the analyses, state so OR if data were excluded, provide the exact number of exclusions and the rationale behind them, indicating whether exclusion criteria were pre-established. 


\section{Ecological, evolutionary \& environmental sciences study design}

All studies must disclose on these points even when the disclosure is negative.

Study description

Research sample

Sampling strategy

Data collection

Timing and spatial scale

Data exclusions

Reproducibility

Randomization

Blinding

Did the study involve field work?

We did not perform randomization as our study was observational rather than experimental.

Each sequenced S. aureus genome was subjected to multilocus sequence typing and the derived clonal complex was compared to the spa type of the original isolate when available, including 63\% (154/244) of the isolates from the hedgehog survey and 63\% (576/913), of the isolates from other sources. The analysis showed that the two methods assigned 100\% (730/730) of the isolates to the same clonal complex. Determination of T. erinacei inhibition zones against the panel of S. aureus strains was repeated four times for each strain. The dates of the most recent common ancestors of the major mecC-MRSA lineages were analysed by two different approaches (BactDating and Treedater), which produced very similar estimates.

skin, and feet of hedgehogs in connection with routine checks. The survey included 828 samples from 276 hedgehogs originating from 16 wild life rescue centres in ten European countries and two wildlife rescue centres in New Zealand, which represent the geographic distribution of the two European hedgehog species, the west European hedgehog (Erinaceus europaeus) present in Western Europe and New Zealand and the northern white-breasted hedgehog (E. roumanicus) found in Eastern Europe. Of the 276 hedgehogs included in the survey, 251 belonged to E. europaeus and the remaining 25 to E. roumanicus. Determination of sex and age was not relevant and therefore not determined. This led to the identification of 244 S. aureus isolates. The survey is a convenience sample, because the number and geographic distribution of hedgehog samples were limited by the number of wildlife rescue centres that volunteered to participate in our study and the number of hedgehogs in care at the time of sampling. We also included a collection of $913 \mathrm{~S}$. aureus CC130, CC425, and CC1943 isolates from other sources, which represents the known geographic distribution (mainly Western and Central Europe) and host repertoire (mainly humans, cattle, sheep, goats, and wild animals) of each clone.

We did not use statistical methods to predetermine sample size, because the number and geographic distribution of hedgehog samples were limited by the number of wildlife rescue centres that volunteered to participate in our study and the number of hedgehogs in care at the time of sampling. S. aureus CC130, CC425, and CC1943 isolates from other sources were selected to represent the known geographic distribution (mainly Western and Central Europe) and host repertoire (mainly humans, cattle, sheep, goats, and wild animals) of each clone.

Personnel at the different wildlife rescue centres were instructed to obtain samples from the nasal area, skin, and feet of hedgehogs to the National Reference Laboratory for Antimicrobial Resistance at Statens Serum Institut in Denmark or the Department of Veterinary Medicine at University of Cambridge in the United Kingdom immediately after sample collection.

The hedgehog survey was conducted between 12th April and 21st November 2018 and included 16 wildlife rescue centres in ten European countries and two wildlife rescue centres in New Zealand, which represent the geographic distribution of the two European hedgehog species: Attica, Greece, Județul Cluj, Romania, Treviso, Italy, Prague, Czech Republic, Jutland, Denmark, Zealand, Denmark, Valencia, Spain, Catalunya, Spain, Norte Region, Portugal, Loire-sur-Rhône, France, Fouencamps, France, Cornwall, England, Berkshire, England, Cambridgeshire, England, Northumberland, England, Powys, Wales, South Island, New Zealand, and North Island, New Zealand. Swab samples were typically collected within 24 to 48 hours at each wildlife rescue centre. There were no specific instructions on when to collect swab samples, because S. aureus colonisation is not expected to display substantial seasonal fluctuations. The 913 S. aureus CC130, CC425, and CC1943 isolates from other sources were collected between 1975 and 2018 and originated from 16 European countries, which represent the geographic distribution of each clone: Denmark, England, Germany, Spain, Scotland, Sweden, France, Norway, Italy, Austria, Wales, Belgium, Switzerland, Finland, Greenland, and the Netherlands.

No animals, samples, or isolates were excluded from our analyses.

Field work, collection and transport

Field conditions

Describe the study conditions for field work, providing relevant parameters (e.g. temperature, rainfall).

Location

State the location of the sampling or experiment, providing relevant parameters (e.g. latitude and longitude, elevation, water depth). 


\title{
Reporting for specific materials, systems and methods
}

We require information from authors about some types of materials, experimental systems and methods used in many studies. Here, indicate whether each material, system or method listed is relevant to your study. If you are not sure if a list item applies to your research, read the appropriate section before selecting a response.

\begin{tabular}{|c|c|}
\hline$n / a$ & Involved in the study \\
\hline Х & Antibodies \\
\hline Х & Eukaryotic cell lines \\
\hline Х & $\square$ Palaeontology and archaeology \\
\hline$\square$ & $\bigotimes$ Animals and other organisms \\
\hline$\bigotimes$ & Human research participants \\
\hline Х & Clinical data \\
\hline Х & Dual use research of concern \\
\hline
\end{tabular}

\author{
Methods \\ $\mathrm{n} / \mathrm{a}$ Involved in the study \\ Х $\square$ ChIP-seq \\ Х $\square$ Flow cytometry \\ Х $\square$ MRI-based neuroimaging
}

\section{Antibodies}

Antibodies used

Describe all antibodies used in the study; as applicable, provide supplier name, catalog number, clone name, and lot number.

Validation

Describe the validation of each primary antibody for the species and application, noting any validation statements on the manufacturer's website, relevant citations, antibody profiles in online databases, or data provided in the manuscript.

\section{Eukaryotic cell lines}

Policy information about cell lines

Cell line source(s)

Authentication

Mycoplasma contamination

Commonly misidentified lines

(See ICLAC register)

\section{State the source of each cell line used.}

Describe the authentication procedures for each cell line used OR declare that none of the cell lines used were authenticated.

Confirm that all cell lines tested negative for mycoplasma contamination OR describe the results of the testing for mycoplasma contamination OR declare that the cell lines were not tested for mycoplasma contamination.

Name any commonly misidentified cell lines used in the study and provide a rationale for their use.

\section{Palaeontology and Archaeology}

Specimen provenance Provide provenance information for specimens and describe permits that were obtained for the work (including the name of the issuing authority, the date of issue, and any identifying information).

Specimen deposition Indicate where the specimens have been deposited to permit free access by other researchers.

Dating methods

If new dates are provided, describe how they were obtained (e.g. collection, storage, sample pretreatment and measurement), where they were obtained (i.e. lab name), the calibration program and the protocol for quality assurance OR state that no new dates are provided.

Tick this box to confirm that the raw and calibrated dates are available in the paper or in Supplementary Information.

Ethics oversight

Identify the organization(s) that approved or provided guidance on the study protocol, OR state that no ethical approval or guidance was required and explain why not.

Note that full information on the approval of the study protocol must also be provided in the manuscript.

\section{Animals and other organisms}

Policy information about studies involving animals; ARRIVE guidelines recommended for reporting animal research
Laboratory animals
The study did not involve laboratory animals. 

roumanicus) that had been admitted to wildlife rescue centres for treatment of a diverse range of conditions. Determination of sex and age was not relevant and therefore not determined. The sampling procedure did not cause pain, suffering, distress or lasting harm, deprived the animal of usual care, or affected the fate of the animals. The survey did not involve wild hedgehogs captured for scientific purposes.

\section{Field-collected samples The study did not involve samples collected from the field.}

Ethics oversight

The hedgehog survey was conducted by staff members of the wildife rescue centres who collected swab samples in connection with
routine checks. In accordance with the Animal Welfare Act 1999 administered by the New Zealand Ministry for Primary Industries and
Directive 2010/63/EU of the European Parliament and of the Council of 22 September 2010 on the protection of animals used for
scientific purposes, no ethical approval was required as sample collection did not cause pain, suffering, distress or lasting harm
equivalent to, or higher than, that caused by the introduction of a needle in accordance with good veterinary practice or deprived the
animal of usual care. Ethical review was undertaken at the Department of Veterinary Medicine, University of Cambridge (ref. CR76).

Note that full information on the approval of the study protocol must also be provided in the manuscript.

\section{Human research participants}

Policy information about studies involving human research participants

Population characteristics

Describe the covariate-relevant population characteristics of the human research participants (e.g. age, gender, genotypic information, past and current diagnosis and treatment categories). If you filled out the behavioural \& social sciences study design questions and have nothing to add here, write "See above."

Recruitment

Describe how participants were recruited. Outline any potential self-selection bias or other biases that may be present and how these are likely to impact results.

Ethics oversight

Identify the organization(s) that approved the study protocol.
Note that full information on the approval of the study protocol must also be provided in the manuscript.

\section{Clinical data}

Policy information about clinical studies

All manuscripts should comply with the ICMJE guidelines for publication of clinical research and a completed CONSORT checklist must be included with all submissions.

Clinical trial registration Provide the trial registration number from ClinicalTrials.gov or an equivalent agency.

Study protocol Note where the full trial protocol can be accessed OR if not available, explain why.

Data collection Describe the settings and locales of data collection, noting the time periods of recruitment and data collection.

Outcomes

Describe how you pre-defined primary and secondary outcome measures and how you assessed these measures.

\section{Dual use research of concern}

Policy information about dual use research of concern

\section{Hazards}

Could the accidental, deliberate or reckless misuse of agents or technologies generated in the work, or the application of information presented in the manuscript, pose a threat to:
No $\mid$ Yes
$\square$ Public health
$\square \square$ National security
$\square \square$ Crops and/or livestock
$\square \square$ Ecosystems
$\square \square$ Any other significant area 
Experiments of concern

Does the work involve any of these experiments of concern:
No $\mid$ Yes
$\square \square$ Demonstrate how to render a vaccine ineffective
$\square \square$ Confer resistance to therapeutically useful antibiotics or antiviral agents
$\square \square$ Enhance the virulence of a pathogen or render a nonpathogen virulent
$\square \square$ Increase transmissibility of a pathogen
$\square \square$ Alter the host range of a pathogen
$\square \square$ Enable evasion of diagnostic/detection modalities
$\square \square$ Enable the weaponization of a biological agent or toxin
$\square \square$ Any other potentially harmful combination of experiments and agents

ChIP-seq

\section{Data deposition}

Confirm that both raw and final processed data have been deposited in a public database such as GEO.

$\square$ Confirm that you have deposited or provided access to graph files (e.g. BED files) for the called peaks.

Data access links
May remain private before publication.
Files in database submission
Genome browser session
(e.g. $\underline{\operatorname{UCSC})}$
For "Initial submission" or "Revised version" documents, provide reviewer access links. For your "Final submission" document, provide a link to the deposited data.

Provide a list of all files available in the database submission.

Provide a link to an anonymized genome browser session for "Initial submission" and "Revised version" documents only, to enable peer review. Write "no longer applicable" for "Final submission" documents.

\section{Methodology}

Replicates

Describe the experimental replicates, specifying number, type and replicate agreement.

Sequencing depth

Describe the sequencing depth for each experiment, providing the total number of reads, uniquely mapped reads, length of reads and whether they were paired-or single-end.

Antibodies

Describe the antibodies used for the ChIP-seq experiments; as applicable, provide supplier name, catalog number, clone name, and lot number.

Peak calling parameters

Specify the command line program and parameters used for read mapping and peak calling, including the ChIP, control and index files used.

Data quality

Describe the methods used to ensure data quality in full detail, including how many peaks are at FDR 5\% and above 5-fold enrichment.

Software

Describe the software used to collect and analyze the ChIP-seq data. For custom code that has been deposited into a community repository, provide accession details.

\section{Flow Cytometry}

Plots

Confirm that:

$\square$ The axis labels state the marker and fluorochrome used (e.g. CD4-FITC).

$\square$ The axis scales are clearly visible. Include numbers along axes only for bottom left plot of group (a 'group' is an analysis of identical markers).

$\square$ All plots are contour plots with outliers or pseudocolor plots.

$\square$ A numerical value for number of cells or percentage (with statistics) is provided.

Methodology

Sample preparation

Describe the sample preparation, detailing the biological source of the cells and any tissue processing steps used.

Instrument

Identify the instrument used for data collection, specifying make and model number. 
Software

Cell population abundance

Gating strategy

Tick this box to confirm that a figure exemplifying the gating strategy is provided in the Supplementary Information.

Magnetic resonance imaging

Experimental design

Design type

Design specifications

Behavioral performance measures

Acquisition

Imaging type(s)

Field strength

Sequence \& imaging parameters

Area of acquisition

Diffusion MRI

Used

Preprocessing

Preprocessing software

Normalization

Normalization template

Noise and artifact removal

Volume censoring

Statistical modeling \& inference

Model type and settings

Effect(s) tested

Specify type of analysis: Whole brain $\square$ ROI-based $\square$ Both

Statistic type for inference (See Eklund et al. 2016)

Correction to establish that the subjects were performing the task as expected (e.g. mean, range, and/or standard deviation across subjects).

Indicate task or resting state; event-related or block design.
$\begin{aligned} & \text { Specify the number of blocks, trials or experimental units per session and/or subject, and specify the length of each trial } \\ & \text { or block (if trials are blocked) and interval between trials. }\end{aligned}$
$\begin{aligned} & \text { State number and/or type of variables recorded (e.g. correct button press, response time) and what statistics were used } \\ & \text { to establish that the subjects were performing the task as expected (e.g. mean, range, and/or standard deviation across } \\ & \text { subjects). }\end{aligned}$

Specify: functional, structural, diffusion, perfusion.

Specify in Tesla

Specify the pulse sequence type (gradient echo, spin echo, etc.), imaging type (EPI, spiral, etc.), field of view, matrix size, slice thickness, orientation and TE/TR/flip angle.

Not used
State whether a whole brain scan was used OR define the area of acquisition, describing how the region was determined.
Provide detail on software version and revision number and on specific parameters (model/functions, brain extraction, segmentation, smoothing kernel size, etc.).

If data were normalized/standardized, describe the approach(es): specify linear or non-linear and define image types used for transformation $O R$ indicate that data were not normalized and explain rationale for lack of normalization.

Describe the template used for normalization/transformation, specifying subject space or group standardized space (e.g. original Talairach, MNI305, ICBM152) OR indicate that the data were not normalized.

Describe your procedure(s) for artifact and structured noise removal, specifying motion parameters, tissue signals and physiological signals (heart rate, respiration).

Define your software and/or method and criteria for volume censoring, and state the extent of such censoring.
Specify type (mass univariate, multivariate, RSA, predictive, etc.) and describe essential details of the model at the first and second levels (e.g. fixed, random or mixed effects; drift or auto-correlation).

Define precise effect in terms of the task or stimulus conditions instead of psychological concepts and indicate whether ANOVA or factorial designs were used.
Specify voxel-wise or cluster-wise and report all relevant parameters for cluster-wise methods.

Describe the type of correction and how it is obtained for multiple comparisons (e.g. FWE, FDR, permutation or Monte Carlo) 
Models \& analysis

$\mathrm{n} / \mathrm{a}$ Involved in the study

$\square \square$ Functional and/or effective connectivity

$\square \square$ Graph analysis

$\square \square$ Multivariate modeling or predictive analysis

Functional and/or effective connectivity

Graph analysis

Multivariate modeling and predictive analysis
Report the measures of dependence used and the model details (e.g. Pearson correlation, partial correlation, mutual information).

Report the dependent variable and connectivity measure, specifying weighted graph or binarized graph subject-or group-level, and the global and/or node summaries used (e.g. clustering coefficient, efficiency, etc.).

Specify independent variables, features extraction and dimension reduction, model, training and evaluation metrics. 